\title{
Orthogonal Synthesis of "Easy-To-Read" Information-containing Polymers using Phosphoramidite and Radical Coupling Steps
}

\author{
Gianni Cavallo, ${ }^{1}$ Abdelaziz Al Ouahabi, ${ }^{1}$ Laurence Oswald, ${ }^{1}$ Laurence Charles, ${ }^{2 *}$ \\ and Jean-François Lutz ${ }^{1 *}$
}

${ }^{1}$ Precision Macromolecular Chemistry, Institut Charles Sadron, UPR22-CNRS, BP84047, 23 rue du Loess, 67034 Strasbourg Cedex 2, France. Email: jflutz@ unistra.fr. ${ }^{2}$ Aix-Marseille Université, CNRS, UMR 7273, Institute of Radical Chemistry, 13397, Marseille Cedex 20, France. Email: laurence.charles@univ-amu.fr

\section{Content:}

$\begin{array}{ll}\text { A. Experimental procedures } & \text { S2 }\end{array}$

$\begin{array}{ll}\text { A.1. Materials } & \text { S2 }\end{array}$

$\begin{array}{ll}\text { A.2. Monomer synthesis } & \text { S3 }\end{array}$

$\begin{array}{ll}\text { A.2.1. Synthesis of phosphoramidite monomers } & \text { S3 }\end{array}$

A.2.1.1. Synthesis of the intermediate $\mathbf{H O - \mathbf { 0 } _ { \mathbf { a } }}$ S3

A.2.1.2. Synthesis of the intermediate $\mathbf{H O - \mathbf { 0 } _ { \mathbf { c } }}$ S3

A.2.1.3. Synthesis of the intermediate $\mathbf{H O}-\mathbf{1}_{\mathbf{a}} \quad$ S3

A.2.1.4. Synthesis of the intermediate $\mathbf{H O}-\mathbf{1}_{\mathbf{b}} \quad$ S4

A.2.1.5. Synthesis of phosphoramidite monomer $\mathbf{0}_{\mathbf{a}} \quad$ S4

$\begin{array}{ll}\text { A.2.1.6. Synthesis of phosphoramidite monomer } \mathbf{0}_{\mathbf{c}} & \text { S4 }\end{array}$

A.2.1.7. Synthesis of phosphoramidite monomer $\mathbf{1}_{\mathbf{a}} \quad$ S5

A.2.1.8. Synthesis of phosphoramidite monomer $\mathbf{1}_{\mathbf{b}} \quad$ S5

A.2.2 Synthesis of the TEMPO derivative $\mathbf{T}_{\mathbf{2}} \quad$ S5

A.3. Iterative Solid-phase synthesis of oligomers $\quad$ S6

$\begin{array}{ll}\text { A.3.1 General procedure for coupling step }(i) & \text { S6 }\end{array}$

$\begin{array}{ll}\text { A.3.2 General procedure for coupling step (ii) } & \text { S6 }\end{array}$

A.3.3 General procedure cleavage and deprotection (iii) S6

$\begin{array}{ll}\text { B. Measurements and analysis } & \text { S7 }\end{array}$

$\begin{array}{ll}\text { C. Additional Figures } & \text { S8 }\end{array}$

$\begin{array}{ll}\text { Figure S1 } & \text { S8 }\end{array}$

$\begin{array}{ll}\text { Figure } \mathrm{S} 2 & \text { S9 }\end{array}$

$\begin{array}{ll}\text { Figure S3 } & \text { S10 }\end{array}$

$\begin{array}{ll}\text { Figure S4 S11 } & \text { S11 }\end{array}$

\begin{tabular}{ll} 
Figure S5 & S12 \\
\hline
\end{tabular}

$\begin{array}{ll}\text { Figure S6 } & \text { S13 }\end{array}$

$\begin{array}{ll}\text { Scheme S2 } & \text { S14 }\end{array}$

Figure S7 $\quad$ S15

Figure S8 $\quad$ S16

$\begin{array}{ll}\text { Figure S9 } & \text { S17 }\end{array}$

$\begin{array}{ll}\text { Figure S10 } & \text { S18 }\end{array}$

$\begin{array}{ll}\text { Figure S11 } & \text { S19 }\end{array}$

$\begin{array}{ll}\text { D. References } & \text { S20 }\end{array}$ 


\section{A. Experimental procedures}

\section{A.1. Materials}

3-Amino-1-propanol (99\%, Alfa Aesar), 3-amino-2,2-dimethyl-1-propanol (97\%, TCI Europe), 4amino-1-butanol (98\%, TCI Europe), 5-amino-1-pentanol (>95\%, TCI Europe), 4-amino-2methyl-1-butanol (98\%, TCI Europe), 4-amino-TEMPO (97\%, TCI), 2-bromoisobutyryl bromide (97\%, Alfa Aesar), $\varepsilon$-caprolactone (97\%, Sigma-Aldrich), 2-cyanoethyl- $N, N$-diisopropylchlorophosphoramidite $(97 \%, \mathrm{ABCR}), 4,4^{\prime}$-dimethoxytriphenylmethyl chloride ( $\geq 97.0 \%$, SigmaAldrich), N,N-diisopropylethylamine (DIPEA, 99\%, TCI), 4-hydroxy-TEMPO ( $\mathrm{T}_{1}, 98 \%, \mathrm{TCI}$ ), 2,6-lutidine (98\%, Alfa Aesar), tris(2-dimethylaminoethyl)amine (99\%, Alfa Aesar), 1methylimidazole (NMI, 99\%, Alfa Aesar), piperidine (99\%, Alfa Aesar), methylamine (aqueous solution, $40 \%$, Fluka), ammonium hydroxide solution (28-30\% $\mathrm{NH}_{3}$, VWR), tetrazole (acetonitrile solution, 0.45M, Sigma-Aldrich), triethylamine (97\%, Merck), trichloroacetic acid (>99\%, SigmaAldrich), 1,4 dioxane (99.5\%, Sigma-Aldrich), tetrahydrofuran (THF, > 99\%, 250 ppm BHT, Sigma-Aldrich), anhydrous dichloromethane (Sigma-Aldrich), anhydrous acetonitrile (SigmaAldrich) and anhydrous dimethyl sulfoxide (DMSO) were used as received. Anhydrous tetrahydrofuran (THF) was obtained on a dry solvent station GTS100. The phosphoramidite compounds were kept in the freezer at $-18^{\circ} \mathrm{C}$. Oligomers syntheses were performed in an equipped argon/vacuum solid phase extraction glass tube $(12 \mathrm{~mL}$ with frit 3$)$ and stirred with an IKA HS 260 Basic shaker. The iterative synthesis was performed on an aminomethylated-polystyrene resin (1.4 mmol. $\mathrm{g}^{-1}$, Merck) that was modified with 4-oxo-4-(3-(dimethoxy-trityloxy)propoxy)butanoic acid as previously reported. ${ }^{[1]}$

Scheme S1. General strategy used for the synthesis of the coded monomers. ${ }^{[2]}$
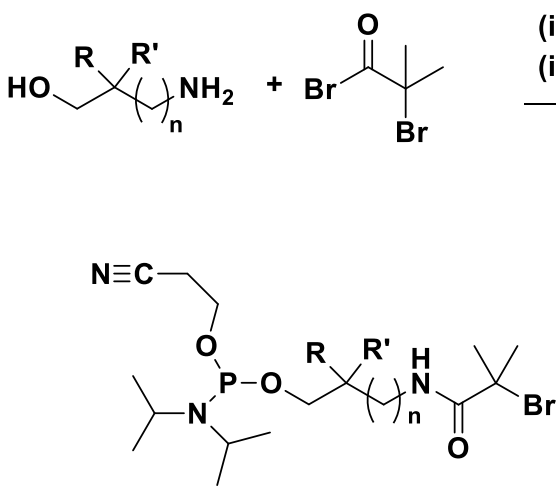

$\mathbf{0}_{\mathbf{a}}:\left(n=1, R, R^{\prime}=H\right), \mathbf{0}_{\mathbf{b}}:\left(n=2, R, R^{\prime}=H\right)$

$\mathbf{0}_{\mathbf{c}}:\left(\mathrm{n}=3, \mathrm{R}, \mathrm{R}^{\prime}=\mathrm{H}\right), \mathbf{1}_{\mathbf{a}}:\left(\mathrm{n}=1, \mathrm{R}, \mathrm{R}^{\prime}=\mathrm{CH}_{3}\right)$

$1_{b}:\left(n=2, R=C_{3}, R^{\prime}=H\right)$
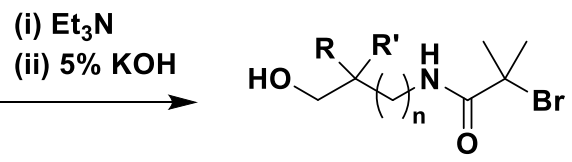

HO- $\mathbf{O}_{\mathrm{a}}:\left(\mathrm{n}=1, \mathrm{R}, \mathrm{R}^{\prime}=\mathrm{H}\right)$

HO- $\mathbf{O}_{\mathrm{b}}:\left(n=2, R, R^{\prime}=H\right)$

HO- $\mathbf{c}_{\mathrm{c}}:\left(\mathrm{n}=3, \mathrm{R}, \mathrm{R}^{\prime}=\mathrm{H}\right)$

HO-1 $\mathbf{a}^{\mathrm{a}}:\left(\mathrm{n}=1, \mathrm{R}, \mathrm{R}^{\prime}=\mathrm{CH}_{3}\right)$

HO-1 $\mathbf{b}:\left(n=2, R=\mathrm{CH}_{3}, \mathrm{R}^{\prime}=\mathrm{H}\right)$

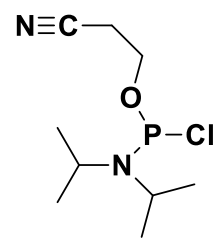

DCM, DIPEA, NMI 


\section{A.2. Monomer synthesis}

\section{A.2.1. Synthesis of the phosphoramidite monomers}

Monomer 0 $\mathbf{b}$ was synthesized according to a procedure from the literature. ${ }^{[2]}$ The other phosphoramidite monomers $\mathbf{0}_{\mathbf{a}}, \mathbf{0}_{\mathbf{c}}, \mathbf{1}_{\mathbf{a}}$ and $\mathbf{1}_{\mathbf{b}}$ were prepared following the strategy shown in scheme S1 that was adapted from the same article. ${ }^{[2]}$

\section{A.2.1.1. Synthesis of the intermediate HO-0a}

3-Amino-1-propanol (5.0 g, $66.6 \mathrm{mmol}, 1$ eq.) was dissolved in $50 \mathrm{~mL}$ of anhydrous dichloromethane, the solution was cooled to $0{ }^{\circ} \mathrm{C}$ under an argon atmosphere. Triethylamine $\left(10.21 \mathrm{~mL}, 73.2 \mathrm{mmol}, 1.1 \mathrm{eq}\right.$.) was added to the reaction mixture and stirred at $0{ }^{\circ} \mathrm{C}$ for $15 \mathrm{~min}$, 2-bromoisobutyryl bromide ( $8.23 \mathrm{~mL}, 66.6 \mathrm{mmol}, 1$ eq.) was added dropwise to the solution. The reaction mixture was let to reach room temperature and stirred for $1.5 \mathrm{~h}$. The precipitate was removed and the filtrate was stirred with $20 \mathrm{~mL}$ of $5 \% \mathrm{KOH}$ aqueous solution for $1 \mathrm{~h}$. The mixture was poured in a separatory funnel and the aqueous phase was separated. The organic layer was washed with $\mathrm{NaOH} 1 \mathrm{M}(2 \times 35 \mathrm{~mL}), \mathrm{HCl} 1 \mathrm{M}(2 \times 35 \mathrm{~mL})$, brine $(1 \times 40 \mathrm{~mL})$, dried over $\mathrm{Na}_{2} \mathrm{SO}_{4}$ and evaporated. The product HO-0a was recovered as a pale yellow oil in $80 \%$ yield. ${ }^{1} \mathrm{H}$ NMR

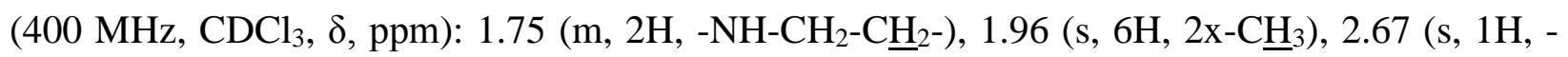

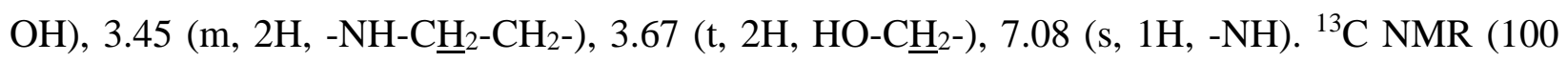

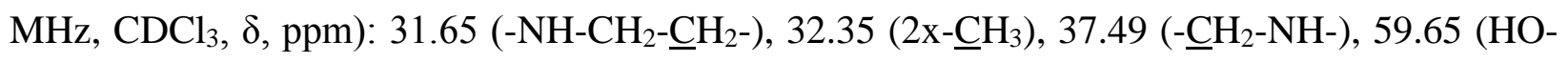
$\left.\underline{\mathrm{CH}}_{2}-\right), 62.18\left(-\underline{\mathrm{C}}-\left(\mathrm{CH}_{3}\right)_{2} \mathrm{Br}\right), 172.95(-\underline{\mathrm{C}}=\mathrm{O}-\mathrm{NH}-)$.

\section{A.2.1.2. Synthesis of the intermediate HO-0c}

The product was recovered as a pale yellow oil in $84 \%$ yield. ${ }^{1} \mathrm{H}$ NMR $\left(400 \mathrm{MHz}, \mathrm{CDCl}_{3}, \delta\right.$, ppm): $1.42\left(\mathrm{~m}, 2 \mathrm{H},-\mathrm{NH}-\mathrm{CH}_{2}-\mathrm{CH}_{2}-\mathrm{C}_{2}-\right), 1.59$ (m, 5H, -NH-CH $\left.2-\underline{\mathrm{C}}_{2}-\mathrm{CH}_{2}-\mathrm{C}_{2}-,-\mathrm{OH}\right), 1.95$ (s, 6H, 2x$\left.\mathrm{C}_{3}\right), 3.28$ (m, 2H, -NH-C $\left.\underline{H}_{2}-\mathrm{CH}_{2}-\right), 3.66$ (t, $\left.2 \mathrm{H}, \mathrm{HO}-\underline{\mathrm{CH}}_{2}-\right), 6.75$ (s, $\left.1 \mathrm{H},-\mathrm{NH}\right) .{ }^{13} \mathrm{C}$ NMR (100

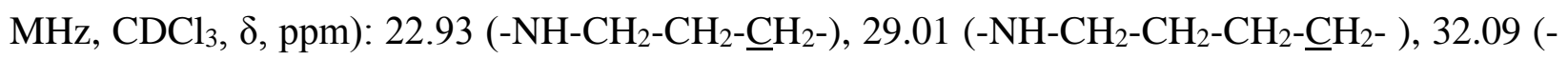
$\left.\mathrm{NH}-\mathrm{CH}_{2}-\underline{\mathrm{CH}}_{2}-\right), 32.53\left(2 \mathrm{x}-\underline{\mathrm{CH}}_{3}\right), 40.20\left(-\underline{\mathrm{CH}}_{2}-\mathrm{NH}-\right), 62.44\left(\mathrm{HO}-\mathrm{CH}_{2}-\right), 63.23\left(-\underline{\mathrm{C}}-\left(\mathrm{CH}_{3}\right)_{2} \mathrm{Br}\right)$, $171.99(-\underline{\mathrm{C}}=\mathrm{O}-\mathrm{NH}-)$.

\section{A.2.1.3. Synthesis of the intermediate HO-1a}

The product was recovered as a white solid in $74 \%$ yield after recrystallization in ethyl acetate/ $n$ pentane. ${ }^{1} \mathrm{H}$ NMR (400 MHz, $\mathrm{CDCl}_{3}, \delta$, ppm): 0.90 (s, 6H, - $\left.\mathrm{CH}_{2}-\mathrm{C}-\left(\mathrm{C}_{3}\right)_{2}\right), 1.96$ (s, 6H, 2x-C $\left.\underline{H}_{3}\right)$,

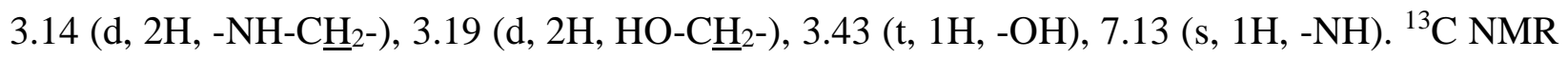


(100 MHz, $\mathrm{CDCl}_{3}, \delta$, ppm): $22.53\left(-\mathrm{CH}_{2}-\mathrm{C}-\left(\underline{\mathrm{CH}}_{3}\right)_{2}\right), 32.52\left(2 \mathrm{x}-\underline{\mathrm{CH}}_{3}\right), 36.80\left(-\mathrm{NH}-\mathrm{CH}_{2}-\underline{\mathrm{C}}-\right), 47.30$ (-NH- $\left.\underline{\mathrm{CH}}{ }_{2}-\mathrm{C}-\right), 62.50$ (-ㄷ- $\left.\left(\mathrm{CH}_{3}\right)_{2} \mathrm{Br}\right), 68.62\left(\mathrm{HO}-\underline{\mathrm{CH}}_{2}-\right), 173.54$ (- $\left.\underline{\mathrm{C}}=\mathrm{O}-\mathrm{NH}-\right)$.

\section{A.2.1.4. Synthesis of the intermediate HO-1b}

The product was recovered as a pale yellow oil in $78 \%$ yield. ${ }^{1} \mathrm{H} \mathrm{NMR}\left(400 \mathrm{MHz}, \mathrm{CDCl}_{3}, \delta, \mathrm{ppm}\right)$ : $0.95\left(\mathrm{~d}, 3 \mathrm{H},-\mathrm{CH}-\underline{\mathrm{C}}_{3}\right), 1.46\left(\mathrm{~m}, 1 \mathrm{H},-\underline{\mathrm{C}}_{-}-\mathrm{CH}_{3}\right), 1.71$ (m, 2H, -NH-CH$\left.{ }_{2}-\underline{\mathrm{C}}_{2}-\right), 1.83(\mathrm{~s}, 1 \mathrm{H},-\mathrm{OH})$, 1.94 (s, 6H, 2x- $\left.\mathrm{CH}_{3}\right), 3.24-3.43$ (m, 2H, $\left.-\mathrm{NH}-\underline{\mathrm{C}}_{2}-\mathrm{CH}_{2}-\right), 3.44-3.59$ (m, 2H, HO-C $\left.\underline{H}_{2}-\right), 6.92$ (s, $1 \mathrm{H},-\mathrm{NH}) .{ }^{13} \mathrm{C} \mathrm{NMR}\left(100 \mathrm{MHz}, \mathrm{CDCl}_{3}, \delta, \mathrm{ppm}\right): 16.75\left(-\mathrm{CH}-\underline{\mathrm{CH}}_{3}\right), 32.33\left(2 \mathrm{x}-\underline{\mathrm{CH}}_{3}\right), 32.95(-\mathrm{NH}-$

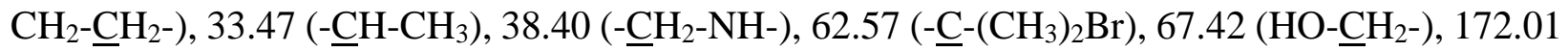
$(-\underline{\mathrm{C}}=\mathrm{O}-\mathrm{NH}-)$.

\section{A.2.1.5. Synthesis of phosphoramidite monomer $0_{a}$}

HO-0a (1.0 g, $4.46 \mathrm{mmol}, 1$ eq.) was dissolved in anhydrous dichloromethane ( $8 \mathrm{~mL}$ ) and DIPEA (3.88 mL, $22.27 \mathrm{mmol}, 5$ eq.) under an argon atmosphere. The solution was stirred at $0{ }^{\circ} \mathrm{C}$ for 5 min, 1-methylimidazole $(0.18 \mathrm{~mL}, 2.23 \mathrm{mmol}, 0.5$ eq. $)$ and a solution of 2-cyanoethyl- $N, N$ diisopropylchlorophosphoramidite (1.16 g, $4.91 \mathrm{mmol}, 1.1 \mathrm{eq}$.) in anhydrous dichloromethane (2 $\mathrm{mL}$ ) were added. The reaction mixture was stirred $30 \mathrm{~min}$ at $0{ }^{\circ} \mathrm{C}$, then was let to reach room temperature and stirred for $1.5 \mathrm{~h}$. The reaction mixture was extracted with saturated $\mathrm{NaHCO}_{3}$ aqueous solution $(12 \mathrm{~mL})$, the organic phase was dried over $\mathrm{Na}_{2} \mathrm{SO}_{4}$ and evaporated. Chromatographic column on silica gel (n-pentane/ethyl acetate/triethylamine) (70/29/1) gives a

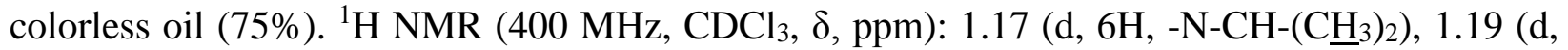

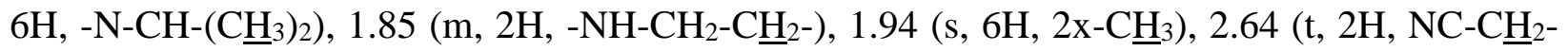

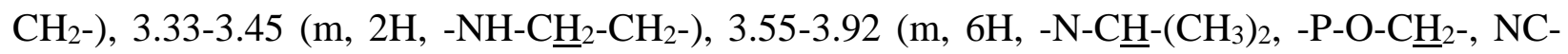
$\left.\mathrm{CH}_{2}-\underline{\mathrm{CH}}_{2}-\right), 6.98$ (s, $\left.1 \mathrm{H},-\mathrm{NH}\right) .{ }^{13} \mathrm{C} \mathrm{NMR}\left(100 \mathrm{MHz}, \mathrm{CDCl}_{3}, \delta, \mathrm{ppm}\right): 20.36,20.44\left(\mathrm{NC}^{-} \mathrm{CH}_{2}-\mathrm{CH}_{2}-\right.$ ), 24.59, 24.65 (-N-CH-( $\left.\left.\underline{\mathrm{CH}}_{3}\right)_{2}\right), 30.27,30.34\left(-\mathrm{NH}-\mathrm{CH}_{2}-\mathrm{CH}_{2}-\right), 32.49$ (2x- $\left.\underline{\mathrm{CH}}_{3}\right), 37.96$ (-NH- ${ }^{-} \mathrm{H}_{2-}$

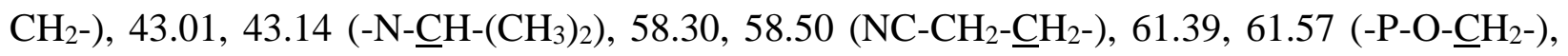
62.80 (- $\left.\underline{\mathrm{C}}-\left(\mathrm{CH}_{3}\right)_{2}-\mathrm{Br}\right), 117.56\left(\mathrm{NC}-\mathrm{CH}_{2}-\mathrm{CH}_{2}-\right), 171.92$ (- $\left.\underline{\mathrm{C}}=\mathrm{O}-\mathrm{NH}-\right) .{ }^{31} \mathrm{P} \mathrm{NMR}\left(161 \mathrm{MHz}, \mathrm{CDCl}_{3}\right.$, $\delta, \mathrm{ppm}): 147.85$.

\section{A.2.1.6. Synthesis of phosphoramidite monomer $0_{c}$}

The product was recovered as a colorless oil in $83 \%$ yield. ${ }^{1} \mathrm{H} \mathrm{NMR}\left(400 \mathrm{MHz}, \mathrm{CDCl}_{3}, \delta, \mathrm{ppm}\right)$ : 1.17 (d, 6H, -N-CH- $\left.\left(\underline{\mathrm{CH}}_{3}\right)_{2}\right), 1.19\left(\mathrm{~d}, 6 \mathrm{H},-\mathrm{N}-\mathrm{CH}-\left(\mathrm{CH}_{3}\right)_{2}\right), 1.42\left(\mathrm{~m}, 2 \mathrm{H},-\mathrm{NH}-\mathrm{CH}_{2}-\mathrm{CH}_{2}-\mathrm{C}_{2}-{ }_{2}\right), 1.59$

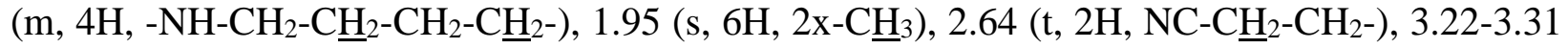

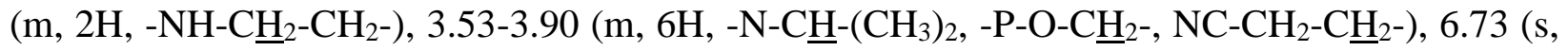


$1 \mathrm{H},-\mathrm{NH}) .{ }^{13} \mathrm{C} \mathrm{NMR}\left(100 \mathrm{MHz}, \mathrm{CDCl}_{3}, \delta, \mathrm{ppm}\right): 20.33,20.39\left(\mathrm{NC}-\mathrm{CH}_{2}-\mathrm{CH}_{2}-\right), 23.30\left(-\mathrm{NH}-\mathrm{CH}_{2}-\right.$ $\left.\mathrm{CH}_{2}-\underline{\mathrm{CH}}_{2}-\right), 24.52,24.58,24.64\left(-\mathrm{N}-\mathrm{CH}-\left(\underline{\mathrm{CH}}_{3}\right)_{2}\right), 28.97$ (-NH-CH$\left.{ }_{2}-\underline{\mathrm{CH}}_{2}-\right), 30.72,30.79$ (-NH-

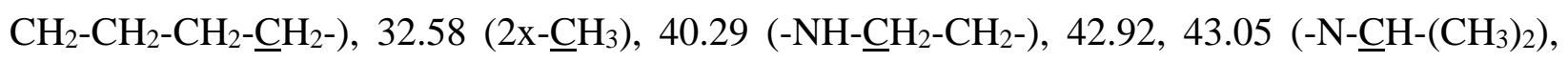

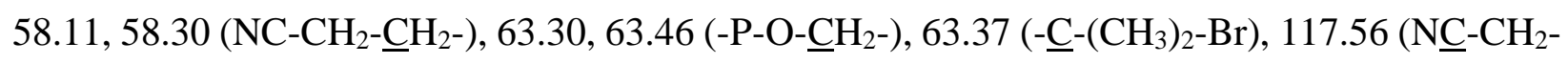
$\left.\mathrm{CH}_{2}-\right), 171.60$ (- $\left.\underline{\mathrm{C}}=\mathrm{O}-\mathrm{NH}-\right) .{ }^{31} \mathrm{P} \mathrm{NMR}\left(161 \mathrm{MHz}, \mathrm{CDCl}_{3}, \delta, \mathrm{ppm}\right): 147.37$.

\section{A.2.1.7. Synthesis of phosphoramidite monomer $1_{\mathrm{a}}$}

The product was recovered as a colorless oil in $90 \%$ yield. ${ }^{1} \mathrm{H} \mathrm{NMR}\left(400 \mathrm{MHz}, \mathrm{CDCl}_{3}, \delta, \mathrm{ppm}\right)$ :

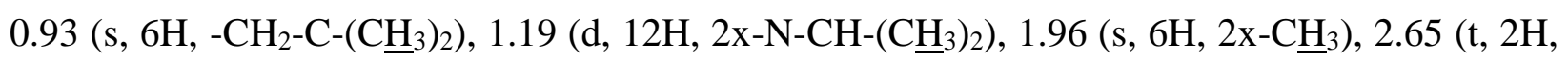
NC-C $\underline{H}_{2}-\mathrm{CH}_{2}-$ ), 3.16-3.26 (m, 2H, -NH-C $\left.\underline{\mathrm{H}}_{2}-\mathrm{C}-\right)$, 3.31-3.46 (m, 2H, -O-C $\underline{\mathrm{H}}_{2}-\mathrm{C}-$ ), 3.56-3.68 (m, $\left.2 \mathrm{H},-\mathrm{N}-\mathrm{C} \underline{\mathrm{H}}-\left(\mathrm{CH}_{3}\right)_{2}\right), 3.78-3.93$ (m, 2H, NC-CH$\left.{ }_{2}-\underline{\mathrm{H}}_{2}-\right), 7.10$ (s, $\left.1 \mathrm{H},-\mathrm{NH}\right) .{ }^{13} \mathrm{C} \mathrm{NMR}(100 \mathrm{MHz}$, $\left.\mathrm{CDCl}_{3}, \delta, \mathrm{ppm}\right): 20.31,20.38\left(\mathrm{NC}-\underline{\mathrm{CH}}_{2}-\mathrm{CH}_{2}-\right), 22.33,22.67\left(-\mathrm{C}-\left(\underline{\mathrm{C}} \mathrm{H}_{3}\right)_{2}\right), 24.49,24.57,24.65$ (-N-

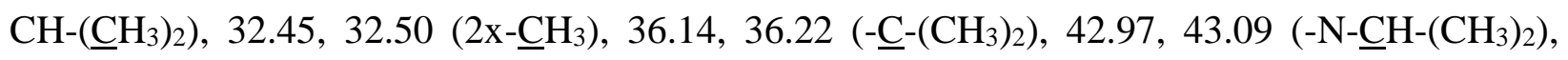
47.70 (-NH- $\left.\underline{\mathrm{CH}}_{2}-\mathrm{C}-\right), 58.20,58.40\left(\mathrm{NC}_{-} \mathrm{CH}_{2}-\underline{\mathrm{CH}}_{2}-\right), 63.01$ (- $\left.\underline{\mathrm{C}}-\left(\mathrm{CH}_{3}\right)_{2}-\mathrm{Br}\right), 70.91,71.06$ (-P-O$\left.\underline{\mathrm{CH}}_{2}-\right), 117.47\left(\mathrm{NC}_{-} \mathrm{CH}_{2}-\mathrm{CH}_{2}-\right), 171.86$ (- $\left.\underline{\mathrm{C}}=\mathrm{O}-\mathrm{NH}-\right) .{ }^{31} \mathrm{P} \mathrm{NMR}\left(161 \mathrm{MHz}, \mathrm{CDCl}_{3}, \delta, \mathrm{ppm}\right)$ : 148.05 .

\section{A.2.1.8. Synthesis of phosphoramidite monomer $1_{b}$}

The product was recovered as a colorless oil in $92 \%$ yield. ${ }^{1} \mathrm{H} \mathrm{NMR}\left(400 \mathrm{MHz}, \mathrm{CDCl}_{3}, \delta, \mathrm{ppm}\right)$ :

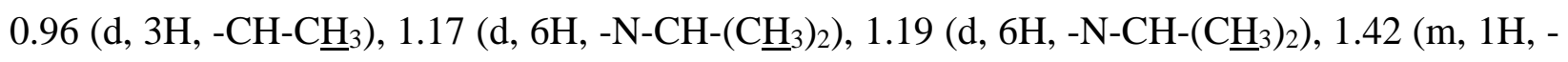

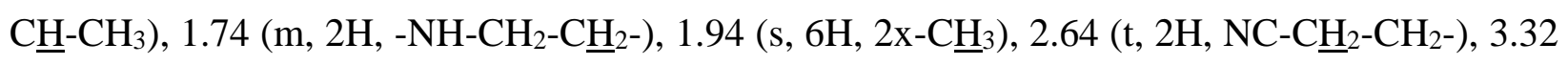

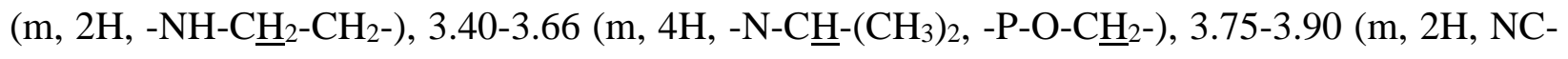
$\left.\mathrm{CH}_{2}-\underline{\mathrm{C}}_{2}-\right), 6.75$ (s, 1H, -NH). ${ }^{13} \mathrm{C} \mathrm{NMR}\left(100 \mathrm{MHz}, \mathrm{CDCl}_{3}, \delta, \mathrm{ppm}\right): 16.76\left(-\mathrm{CH}-\underline{\mathrm{CH}}_{3}\right), 20.32$, $20.39\left(\mathrm{NC}-\underline{\mathrm{CH}}_{2}-\mathrm{CH}_{2}-\right), 24.49,24.51,24.57,24.58$ (-N-CH-( $\left.\left.\underline{\mathrm{CH}}_{3}\right)_{2}\right), 32.36,32.38\left(-\underline{\mathrm{CH}}-\mathrm{CH}_{3}\right)$,

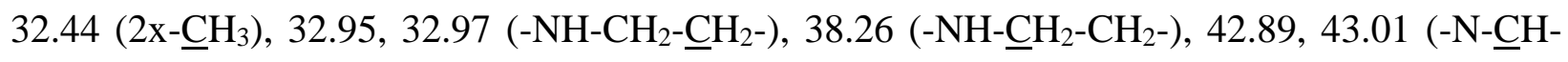

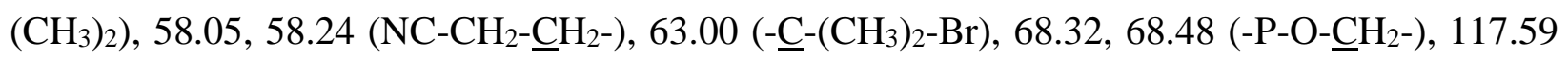
$\left(\mathrm{NC}_{-}-\mathrm{CH}_{2}-\mathrm{CH}_{2}-\right), 171.74$ (-C $\left.=\mathrm{O}-\mathrm{NH}-\right) .{ }^{31} \mathrm{P} \mathrm{NMR}\left(161 \mathrm{MHz}, \mathrm{CDCl}_{3}, \delta, \mathrm{ppm}\right): 147.73$.

\section{A.2.2. Synthesis of the TEMPO derivative $T 2$}

The synthesis of this monomer was adapted from the literature. ${ }^{[3]} 4$-Amino-TEMPO (800 mg, 4.6 mmol) was dissolved in $\varepsilon$-caprolactone (3 eq.) and stirred at $130{ }^{\circ} \mathrm{C}$ for $2 \mathrm{~h}$. The mixture was purified by chromatography on silica gel (dichloromethane/methanol) (95/5) yielding $900 \mathrm{mg}$ of monomer as red oil (69\%). ESI-MS $[\mathrm{M}+\mathrm{H}]^{+}, m / z$ expected for $\mathrm{C}_{15} \mathrm{H}_{30} \mathrm{~N}_{2} \mathrm{O}_{3}$ 286.2, found 287.2. 


\section{A.3. Iterative Solid-phase synthesis of oligomers}

The couplings were carried out under vacuum-argon in rigorously dry conditions using a peptide synthesis reaction vessel equipped with a fritted glass plate and a stopcock.

\section{A.3.1. General procedure for coupling step (i)}

Step $\left(\boldsymbol{i}_{1}\right) .1$ molar equivalent of modified polystyrene resin $(0.8 \mathrm{mmol} / \mathrm{g})$ was placed in the reaction vessel. After dichloromethane swelling, the resin was treated with a $3 \%$ trichloroacetic acid solution in dichloromethane and washed with anhydrous acetonitrile. A phosphoramidite monomer $\left(\mathbf{0}_{\mathbf{a}}, \mathbf{0}_{\mathbf{b}}, \mathbf{0}_{\mathbf{c}}, \mathbf{1}_{\mathbf{a}}\right.$ or $\mathbf{1}_{\mathbf{b}}, 3.3$ eq.) dissolved in anhydrous acetonitrile $(2 \mathrm{~mL})$ and tetrazole (4 eq, $0.45 \mathrm{M}$ solution in acetonitrile) were added under argon atmosphere. The reactor was shaken at room temperature for $1 \mathrm{~h}$. Afterwards, the solution was removed and the resin was washed with acetonitrile. Step $\left(\boldsymbol{i}_{2}\right)$. The resulting phosphite-triester was oxidized into a phosphate-triester via a 20 min treatment with a $0.1 \mathrm{M}$ iodine solution ( $\mathrm{I}_{2}$ in water/2,6-lutidine/THF, 2/20/80). The excess of iodine was removed by repeated anhydrous dichloromethane washing.

\section{A.3.2. General procedure for coupling step (ii)}

After swelling in anhydrous dichloromethane, the resin was washed with anhydrous DMSO. A TEMPO derivative ( $\mathbf{T}_{1}$ or $\mathbf{T}_{2}, 5.5$ eq.) and tris(2-dimethylaminoethyl)amine (3.3 eq.) were dissolved in anhydrous DMSO (2 mL), the solution was added to the vessel under an argon atmosphere, the reactor was shaken at room temperature for 25 minutes. A solution of $\mathrm{CuBr}(3$ eq.) in $2 \mathrm{~mL}$ of anhydrous DMSO was added to the mixture, which was shaken for $1 \mathrm{~h}$. Afterwards, the solution was removed and the polystyrene support was washed several times with THF and 2 times with a $2 \%$ solution of 4,4'-di-n-nonyl-2,2'-dipyridyl in dichloromethane.

\section{A.3.3. General procedure for cleavage and deprotection (iii)}

Before performing step (iii), traces of copper were removed by washing the resin with a solution of $2 \%$ sodium-EDTA in water/THF (1:2 v/v). Deprotection of the cyanoethyl protecting group was done using $10 \%$ piperidine solution in acetonitrile $(2 \times 15 \mathrm{~min})$ afterwards the polymer was cleaved from the resin using a mixture of ammonia aqueous solution, methylamine aqueous solution and 1,4 dioxane $(1: 1: 0.5 \mathrm{v} / \mathrm{v} / \mathrm{v})$ for $1 \mathrm{~h}$ at room temperature. The mixture was filtered and bubbled under argon for $30 \mathrm{~min}$ in order to remove the excess ammonia. The methylamine was removed with a rotavapor and the water by lyophilization. The resulting powders were analyzed by ESI-MS and NMR. Overall yields after cleavage were all in the range 40-60\%. Example of yield for $\alpha$ $0_{\mathrm{b}} \mathrm{T}_{2} 1_{\mathrm{b}} \mathrm{T}_{2} 0_{\mathrm{b}} \mathrm{T}_{2} 0_{\mathrm{b}} \mathrm{T}_{2} 1_{\mathrm{b}} \mathrm{T}_{2} 1_{\mathrm{b}} \mathrm{T}_{2} 1_{\mathrm{b}} \mathrm{T}_{2} \mathrm{O}_{\mathrm{b}} \mathrm{T}_{2}$ (Entry 8 in Table 1): $54 \%$. 


\section{B. Measurements and analysis}

\section{B.1. Nuclear Magnetic Resonance (NMR)}

${ }^{1} \mathrm{H}$ NMR (400 MHz), ${ }^{13} \mathrm{C}$ NMR (100.6 MHz), and ${ }^{31} \mathrm{P}$ NMR (161.92 MHz) spectra were recorded in $\mathrm{CDCl}_{3}$ for monomers and $\mathrm{D}_{2} \mathrm{O}$ for oligomers on a Bruker Avance 400 spectrometer equipped with Ultrashield magnet.

\section{B.1. Mass spectrometry}

High resolution MS and MS/MS experiments were performed using a QqTOF mass spectrometer (QStar Elite, Applied Biosystems SCIEX, Concord, ON, Canada) equipped with an ESI source operated in the negative mode (capillary voltage: $-4200 \mathrm{~V}$; cone voltage: $-75 \mathrm{~V}$ ). In the MS mode, ions were accurately mass measured in the orthogonal acceleration time-of-flight (oa-TOF) mass analyzer, using an internal calibration procedure. In the MS/MS mode, precursor ions were selected in a quadrupole mass analyzer prior entering a collision cell filled with nitrogen, and products ions were measured in the oa-TOF. In this instrument, air was used as nebulizing gas (10 psi) while nitrogen was used as curtain gas (20 psi). Instrument control, data acquisition and data processing were achieved using Analyst software (QS 2.0) provided by Applied Biosystems. All samples were first dissolved in methanol, then diluted with a methanolic solution of ammonium acetate $(3 \mathrm{mM})$ and introduced in the ESI source at a $5 \mu \mathrm{L} \cdot \mathrm{min}^{-1}$ flow rate using a syringe pump. 


\section{Additional data and figures}

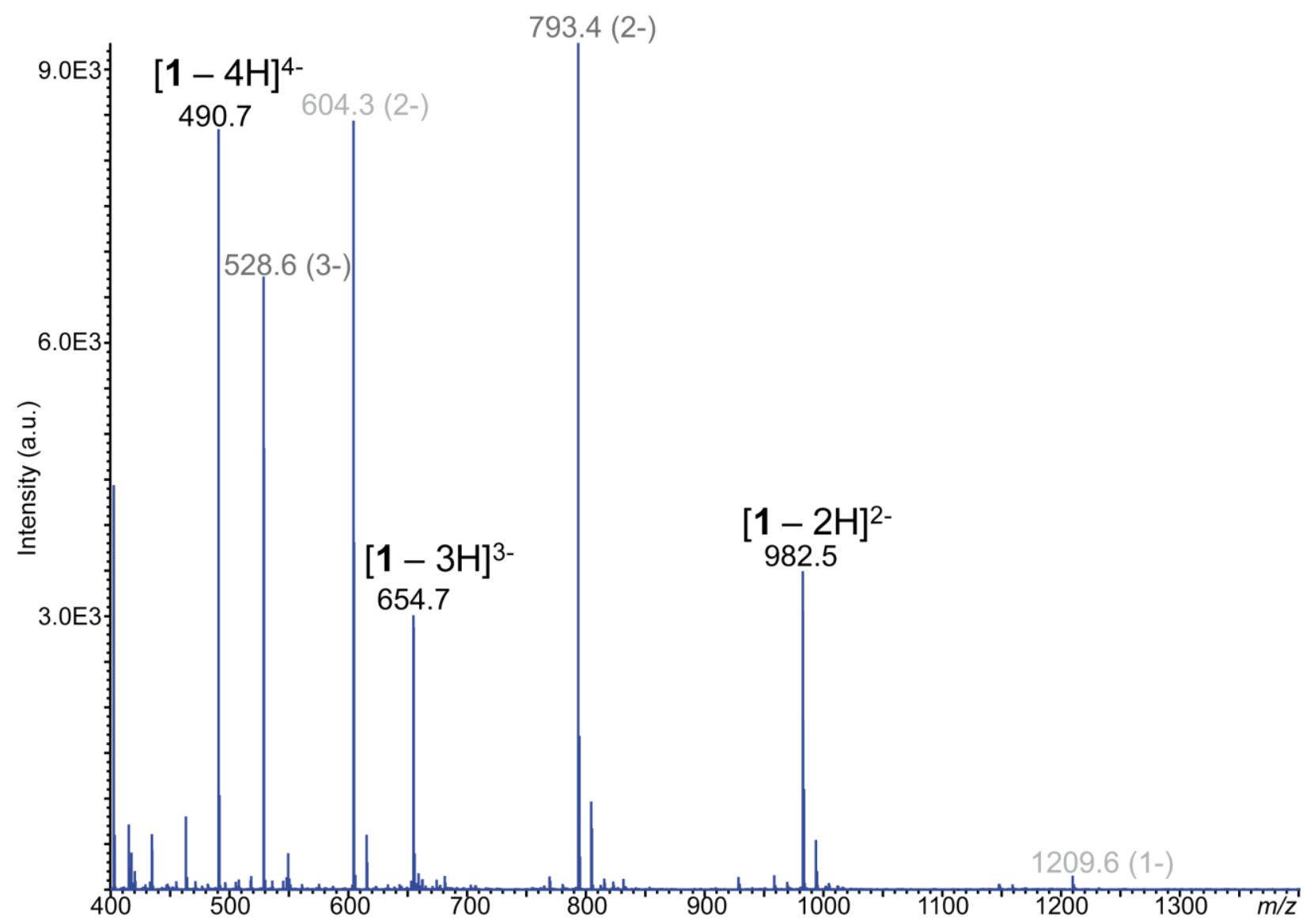

Figure S1. Negative ion mode ESI mass spectrum of $1, \alpha-0_{a} T_{1} 0_{a} T_{1} 0_{a} T_{1} 0_{a} T_{1} 0_{a} T_{1}$. Peaks annotated in grey correspond to deprotonated molecules $[\mathrm{M}-\mathrm{zH}]^{\mathrm{z}-}$ (with $\mathrm{z}$ indicated into parentheses) of lower congeners, $\alpha-0_{\mathrm{a}} \mathrm{T}_{1} 0_{\mathrm{a}} \mathrm{T}_{1} 0_{\mathrm{a}} \mathrm{T}_{1} 0_{\mathrm{a}} \mathrm{T}_{1}$ (dark grey) and $\alpha-0_{\mathrm{a}} \mathrm{T}_{1} 0_{\mathrm{a}} \mathrm{T}_{1} 0_{\mathrm{a}} \mathrm{T}_{1}$ (pale grey). 


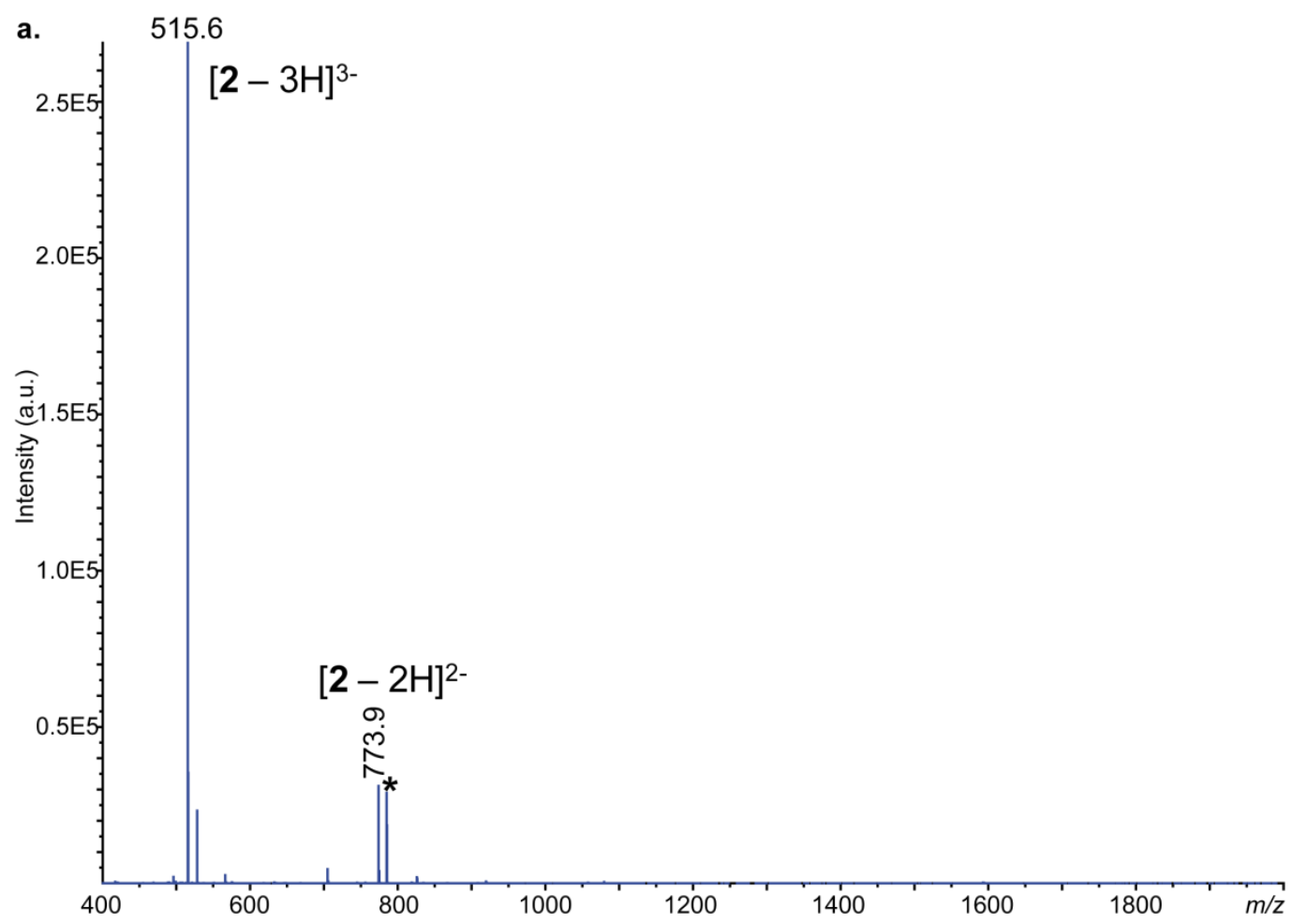

b.
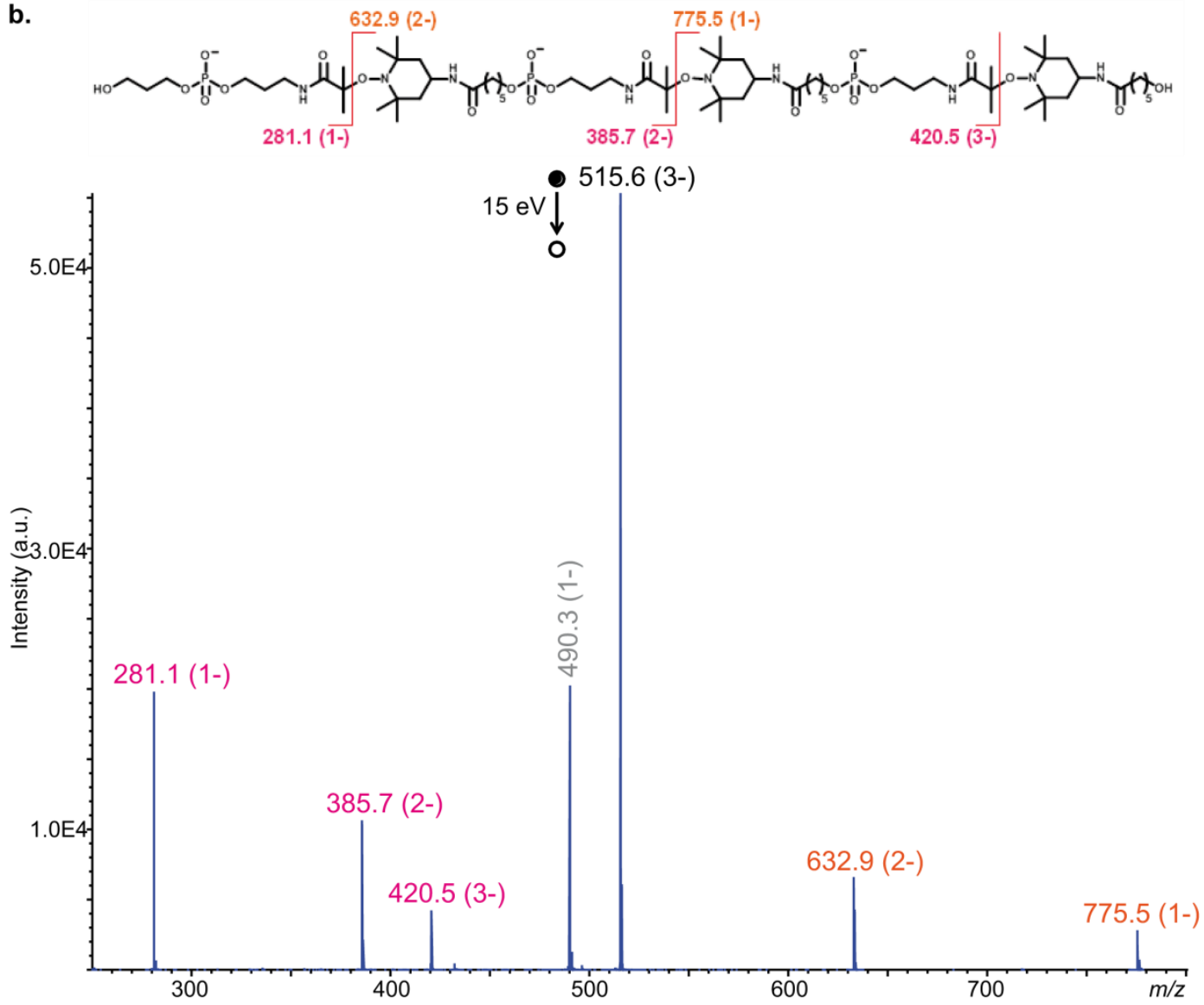

Figure S2. (a) Negative ion mode ESI mass spectrum of 2, $\alpha-0_{a} T_{2} 0_{a} T_{2} 0_{a} T_{2} . *$ designates the ion obtained after $\mathrm{H} / \mathrm{Na}$ exchange, $[2+\mathrm{Na}-3 \mathrm{H}]^{2-}$. (b) MS/MS sequencing of $[2-3 \mathrm{H}]^{3-}$ at $m / z 515.6$ (collision energy : $15 \mathrm{eV}$ ) and corresponding dissociation scheme. 

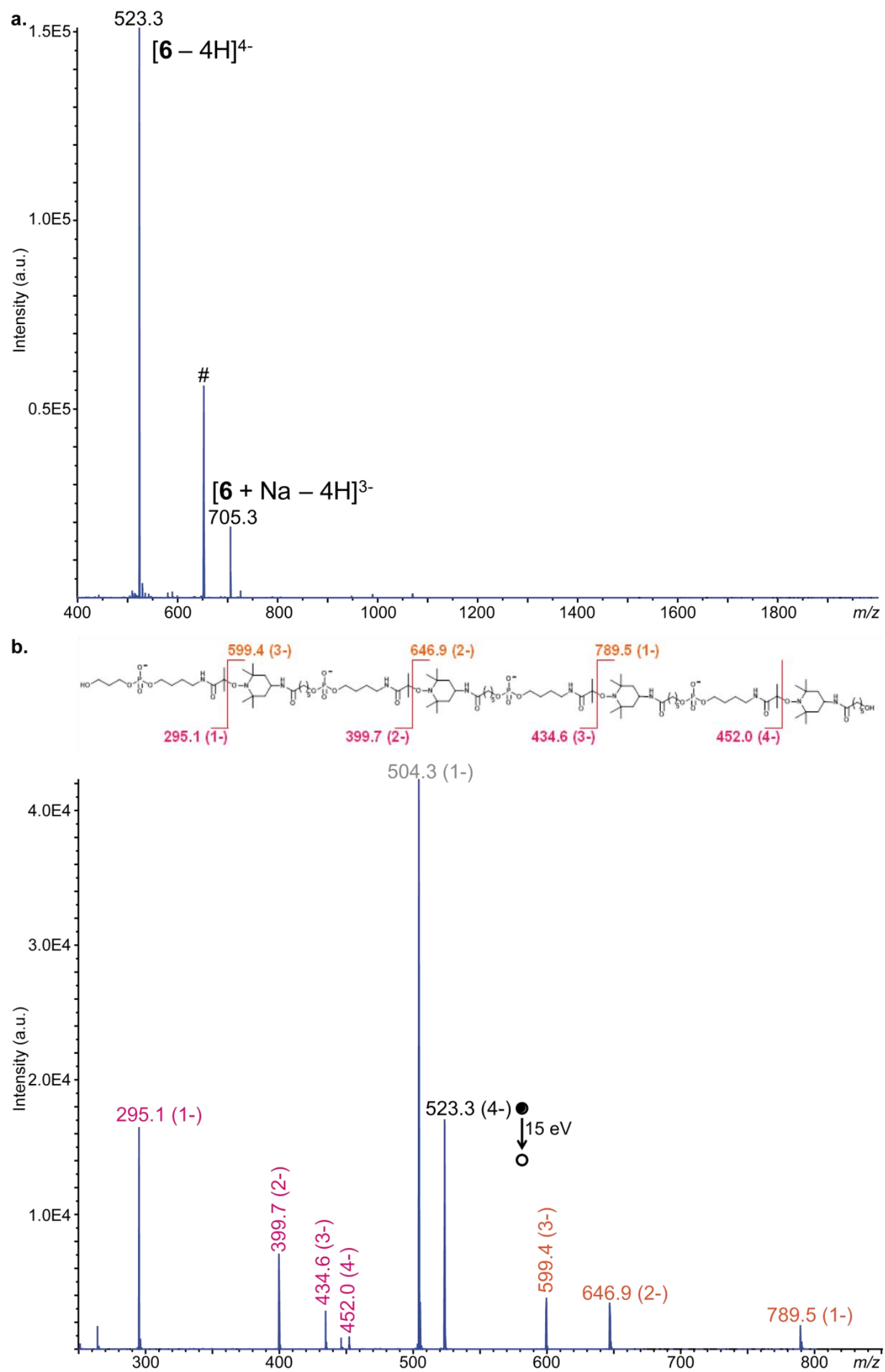

Figure S3. (a) Negative ion mode ESI mass spectrum of 6, $\alpha-0_{b} T_{2} O_{b} T_{2} O_{b} T_{2} O_{b} T_{2}$. \# impurity. (b) MS/MS sequencing of $[6-4 \mathrm{H}]^{4-}$ at $m / z 523.3$ (collision energy : $15 \mathrm{eV}$ ) and corresponding dissociation scheme. 


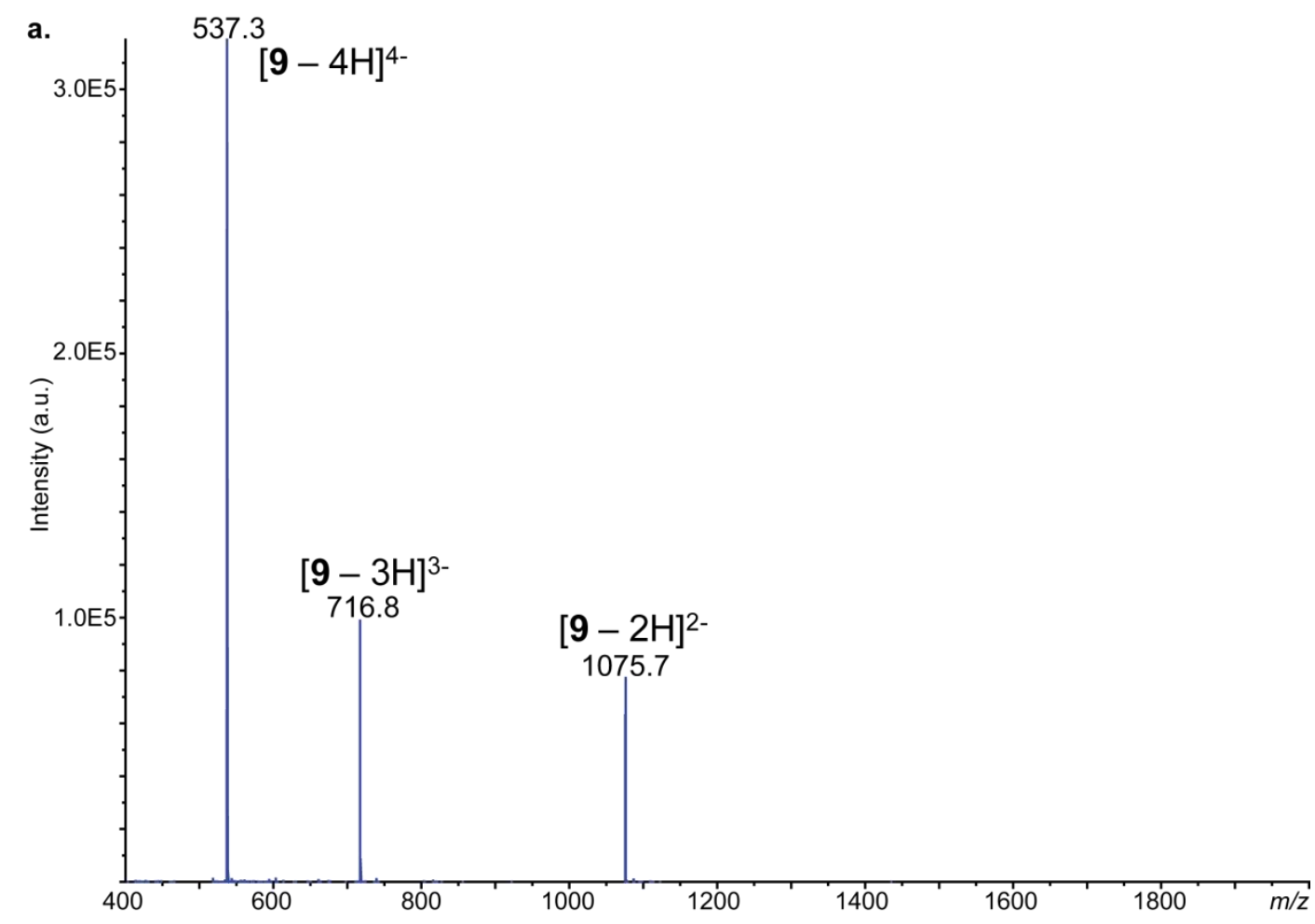

b.
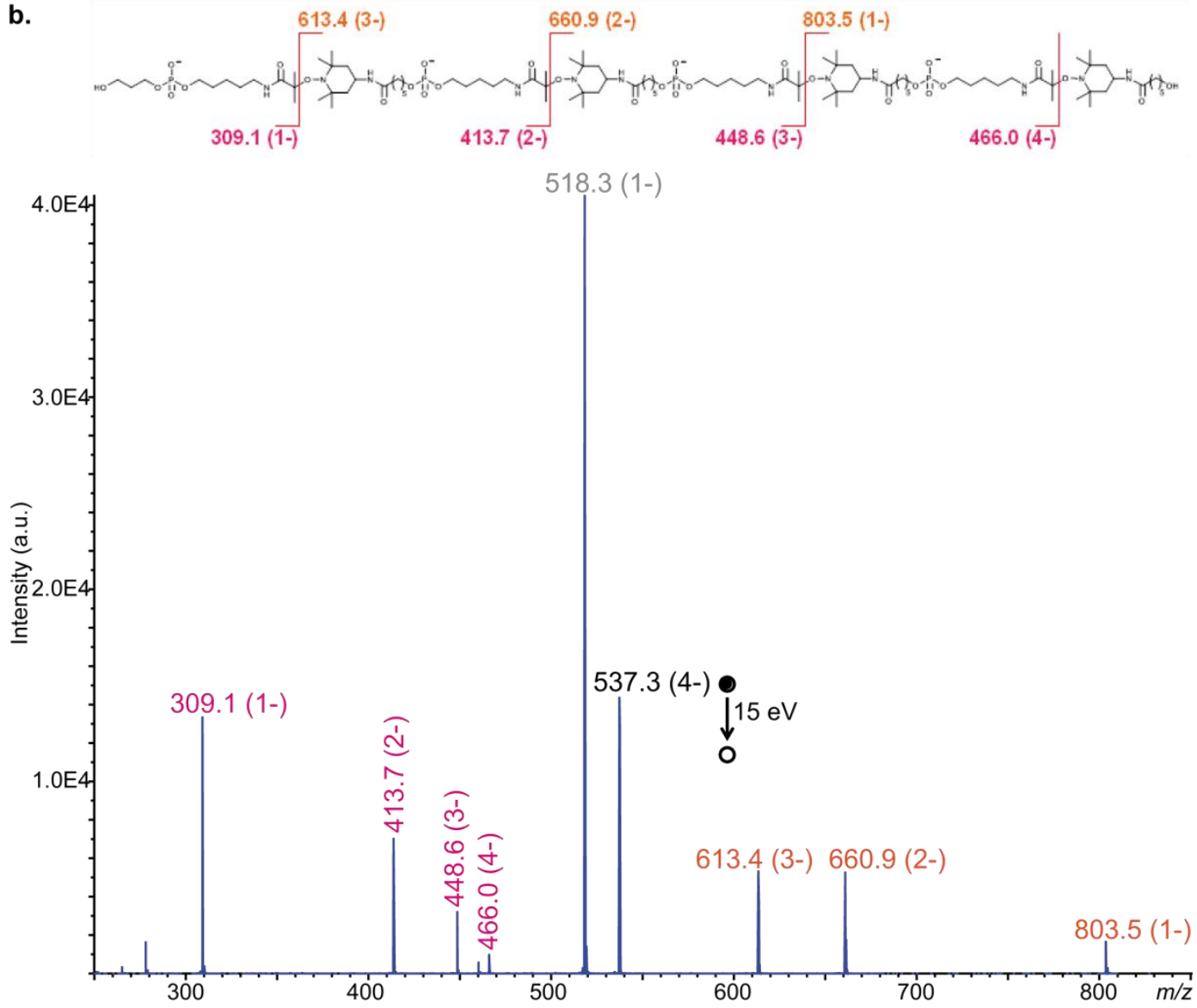

Figure S4. (a) Negative ion mode ESI mass spectrum of 9, $\alpha-0_{c} T_{2} 0_{c} T_{2} 0_{c} T_{2} 0_{c} T_{2}$. (b) MS/MS sequencing of $[9-4 \mathrm{H}]^{4-}$ at $\mathrm{m} / \mathrm{z} 537.3$ (collision energy : $15 \mathrm{eV}$ ) and corresponding dissociation scheme. 
a.
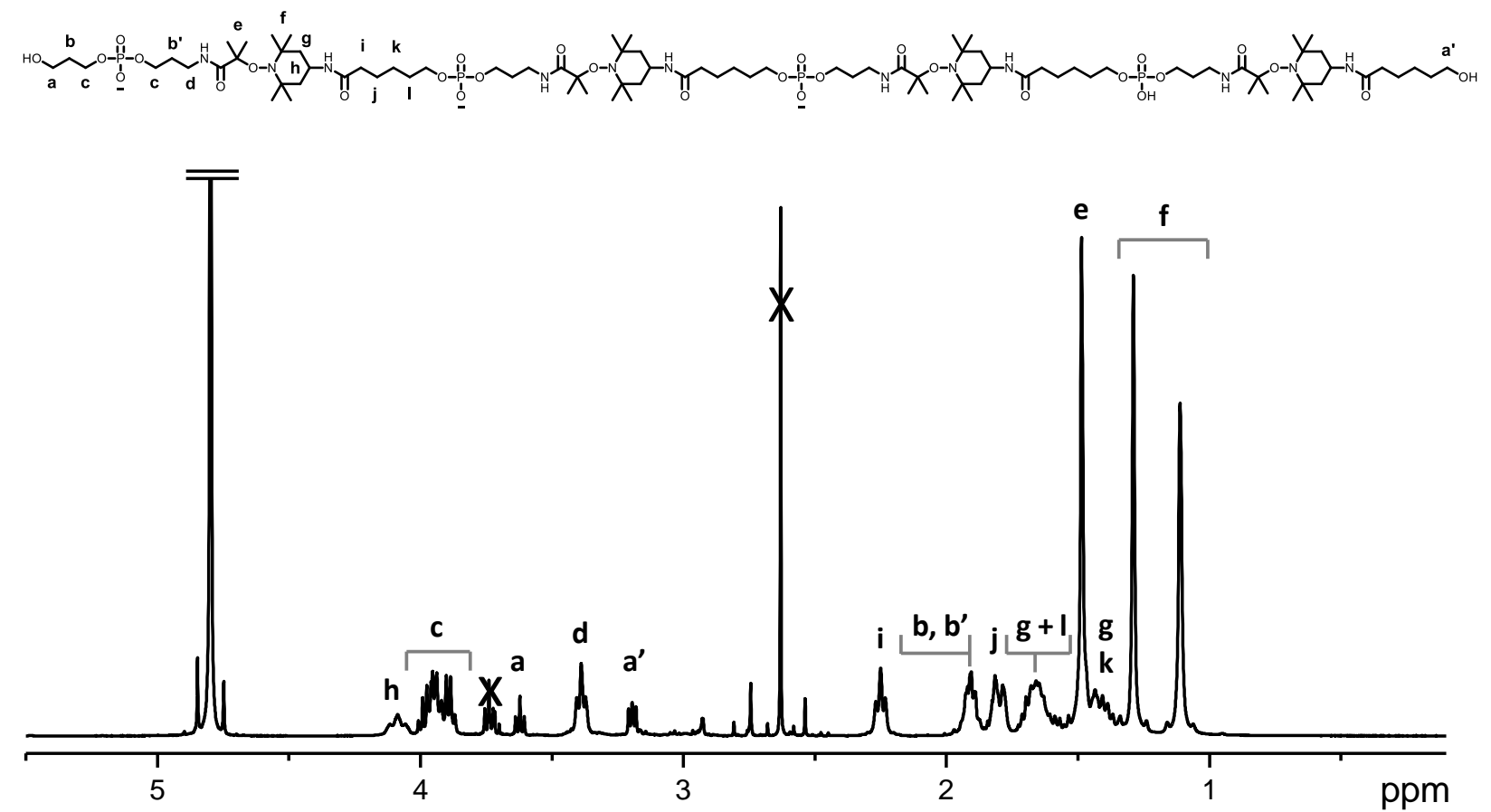

b.
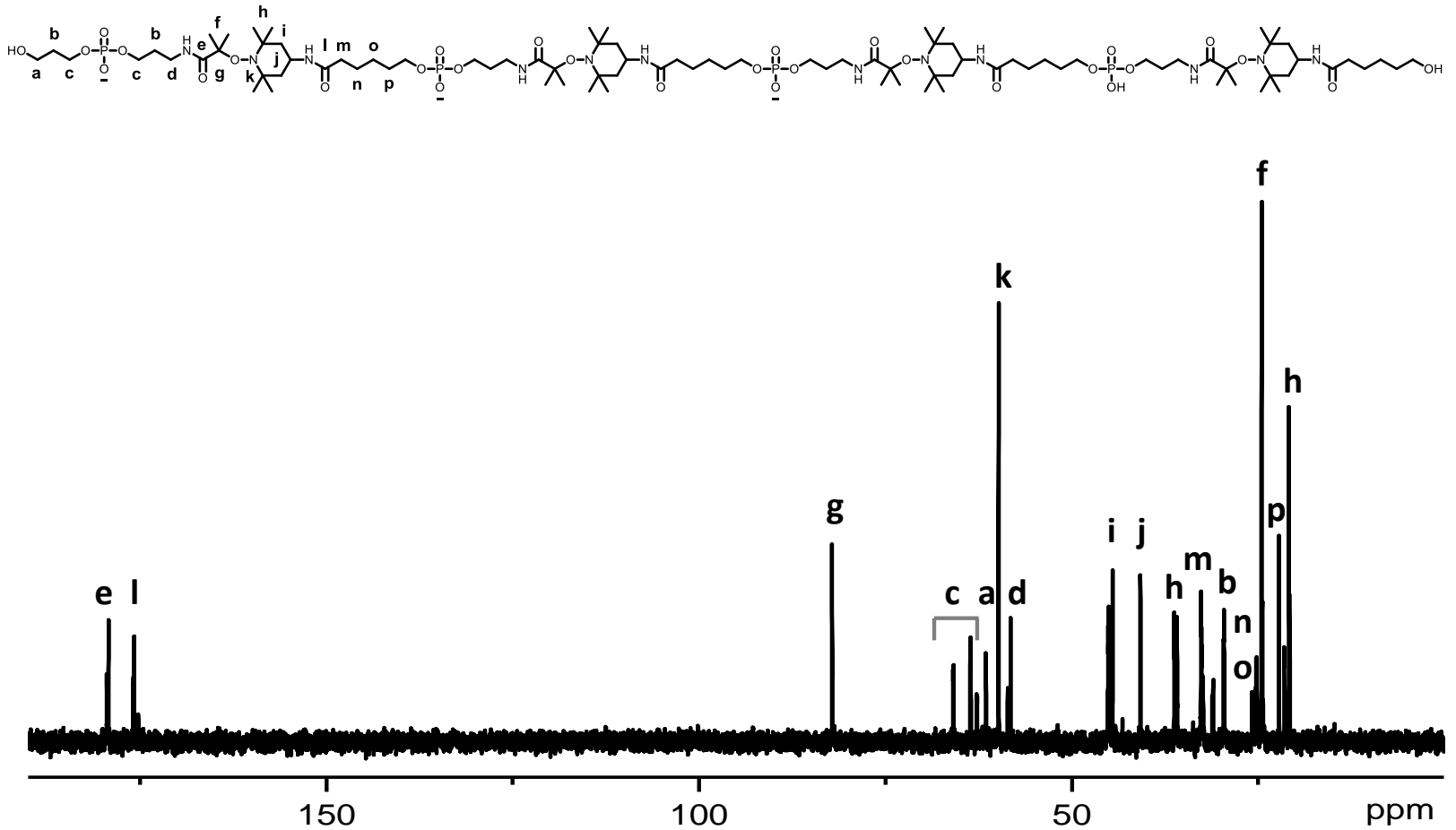

Figure S5. NMR spectra recorded in $\mathrm{D}_{2} \mathrm{O}$ for homopolymer $3 \alpha-0_{\mathrm{a}} \mathrm{T}_{2} \mathrm{O}_{\mathrm{a}} \mathrm{T}_{2} \mathrm{O}_{\mathrm{a}} \mathrm{T}_{2} \mathrm{O}_{\mathrm{a}} \mathrm{T}_{2}$. (a) ${ }^{1} \mathrm{H}$ NMR, (b) ${ }^{13} \mathrm{C}$ NMR. 
a.
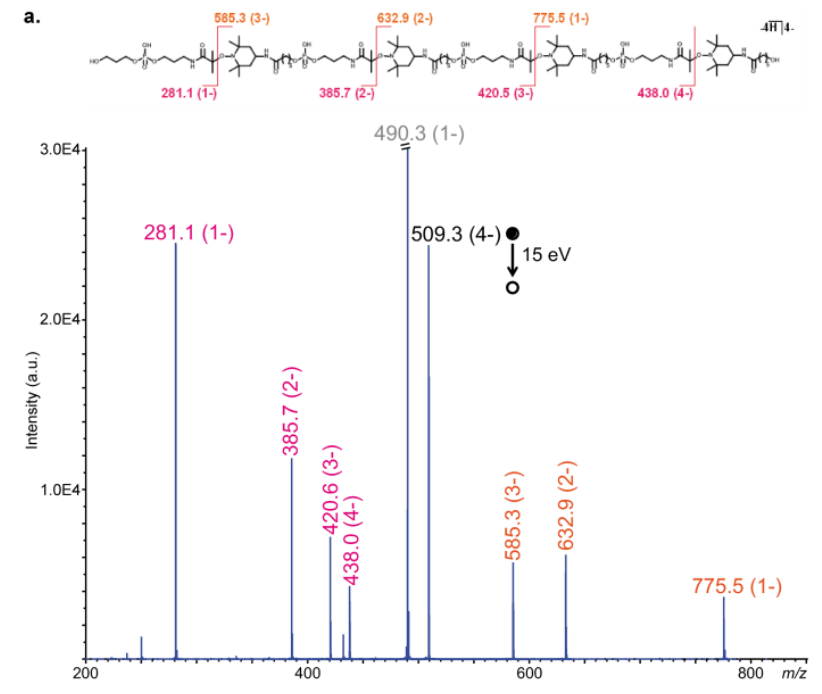

b.
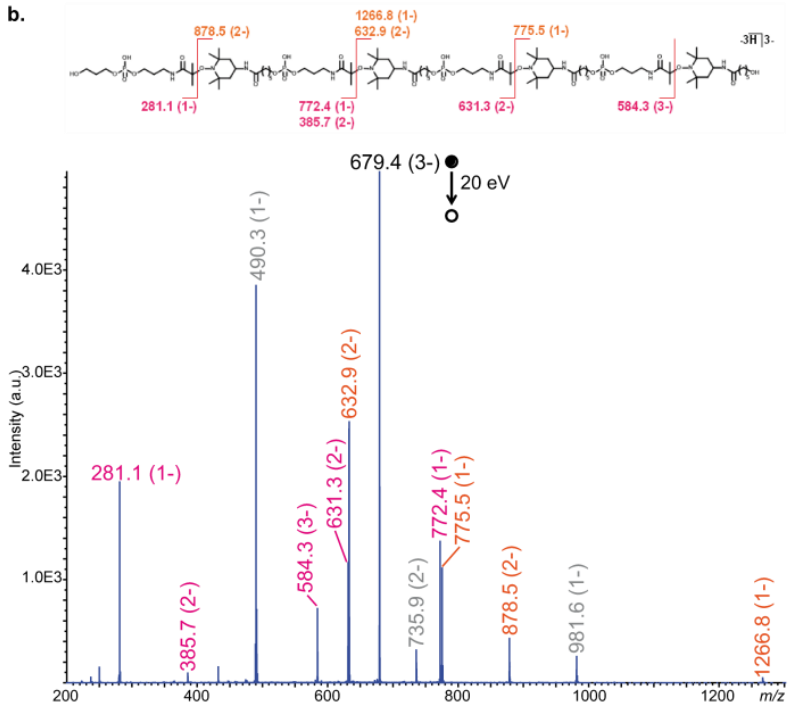

c.
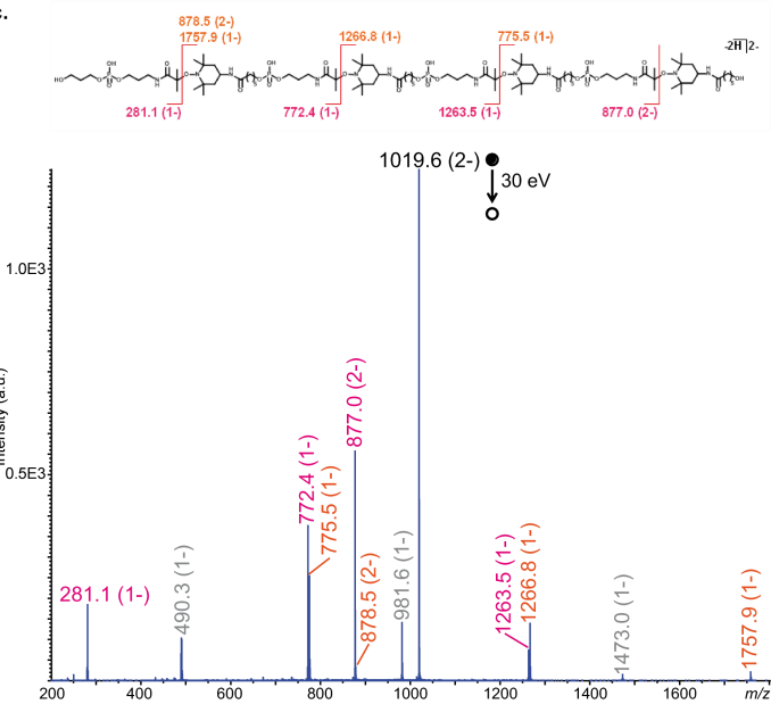

Figure S6. MS/MS sequencing of 3, $\alpha-0_{\mathrm{a}} \mathrm{T}_{2} \mathrm{O}_{\mathrm{a}} \mathrm{T}_{2} \mathrm{O}_{\mathrm{a}} \mathrm{T}_{2} \mathrm{O}_{\mathrm{a}} \mathrm{T}_{2}$, from CID data of (a) $[\mathbf{3}-4 \mathrm{H}]^{4-}$ at $\mathrm{m} / z$ 509.3 (collision energy : $15 \mathrm{eV}),(\mathbf{b})[3-3 \mathrm{H}]^{3-}$ at $\mathrm{m} / z$ 679.4 (collision energy : $20 \mathrm{eV}$ ) and (c) [4$2 \mathrm{H}]^{2-}$ at $\mathrm{m} / \mathrm{z} 1019.6$ (collision energy : $30 \mathrm{eV}$ ), and corresponding dissociation schemes. Peaks designated by $m / z$ values in grey correspond to internal fragments 
Scheme S2. Schematic representation of the different synthons that are detected in MS/MS sequencing. The corresponding light blue, dark blue and black spheres were used in Figures 2 and 3 of the main paper to denote these synthons.
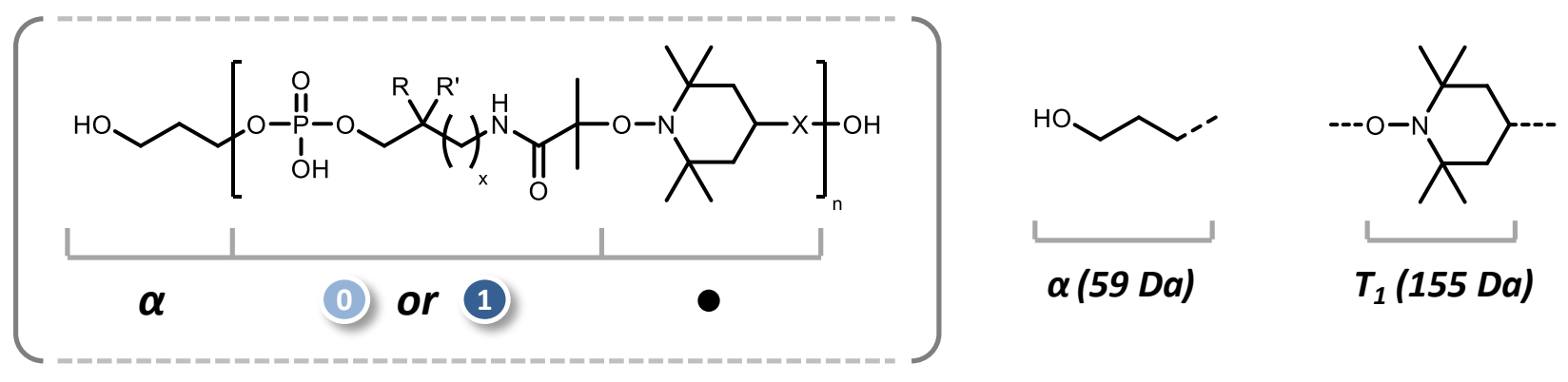

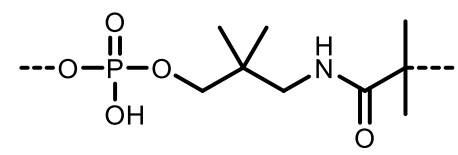

$1_{a}$ motif (251 Da)

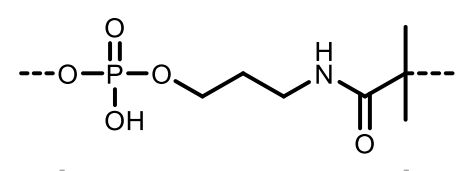

$0_{a}$ motif (223 Da)

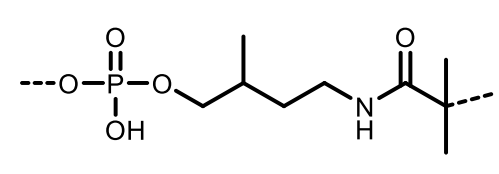

$1_{b}$ motif (251 Da)

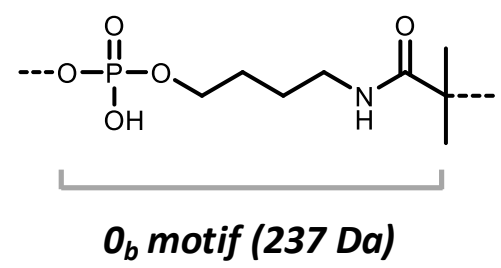

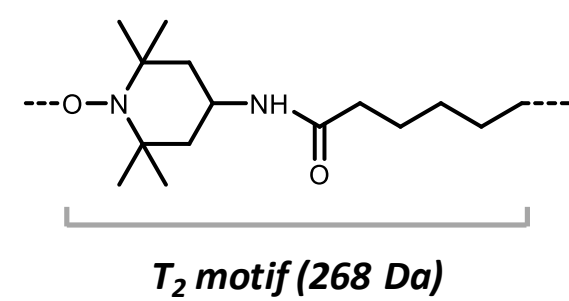

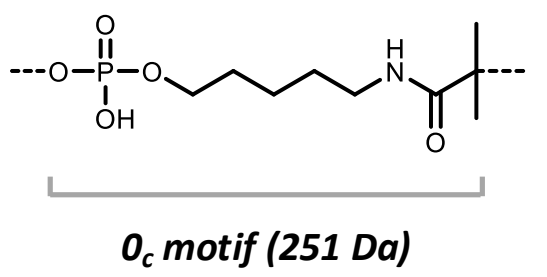




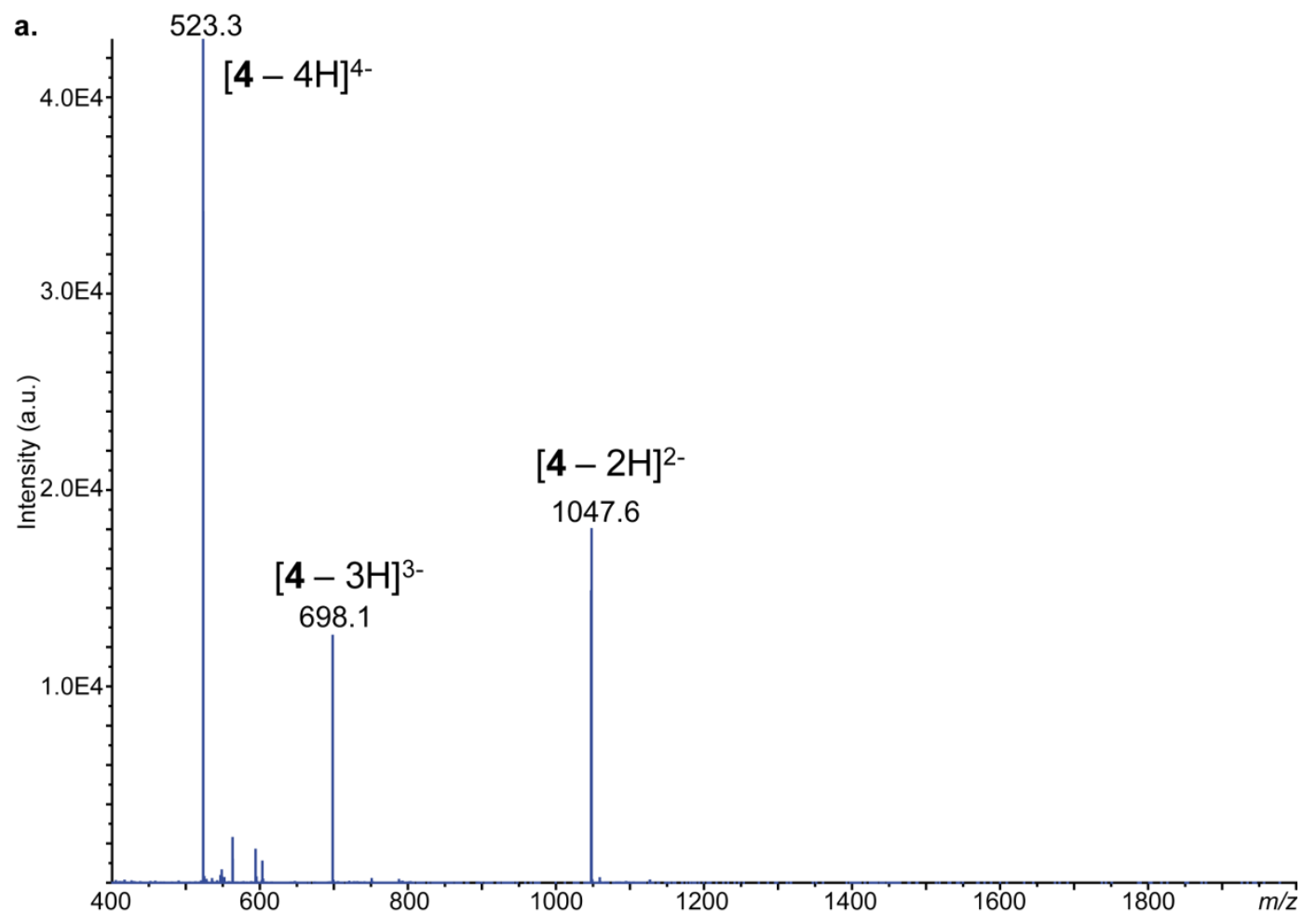

b.
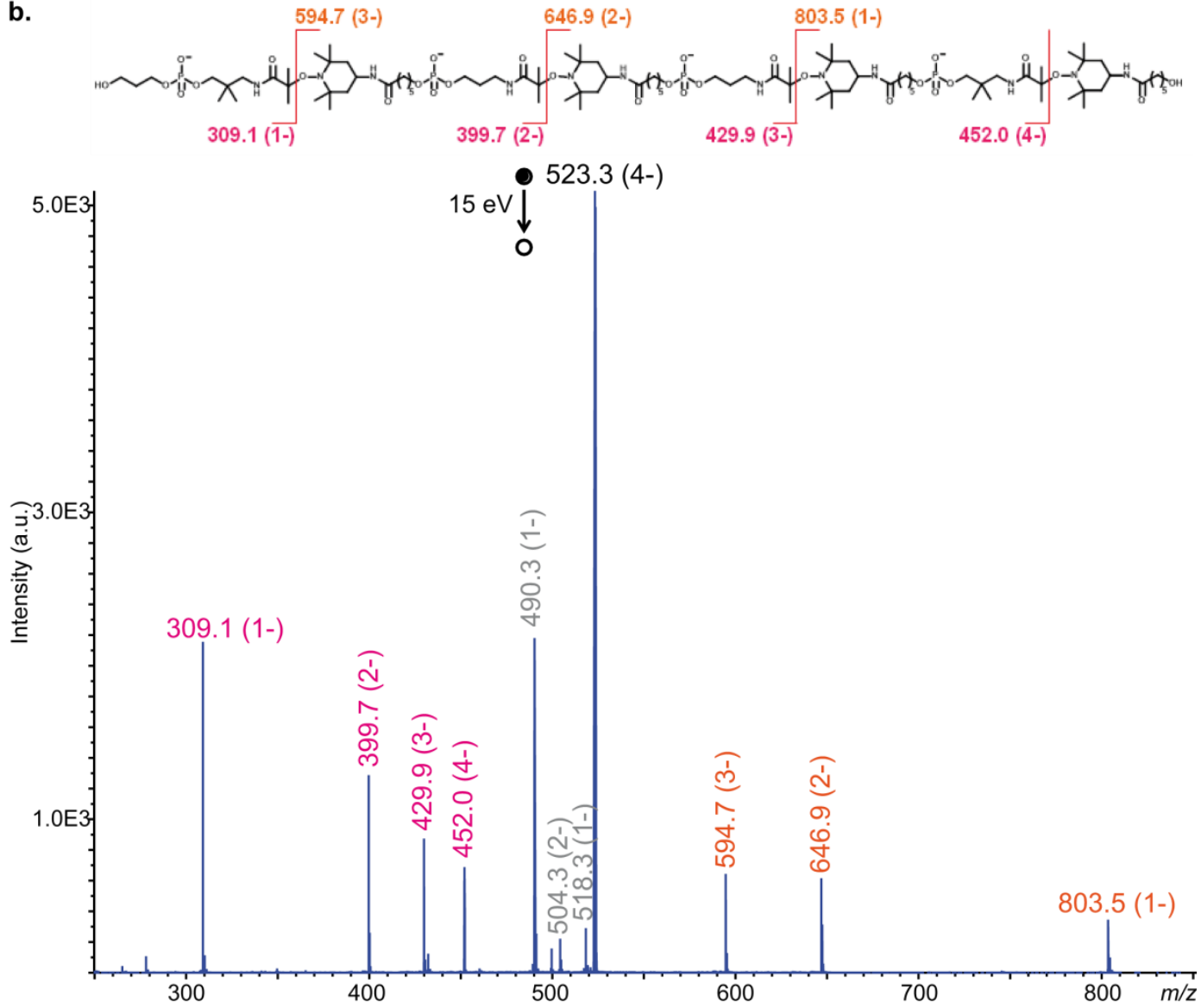

Figure S7. (a) Negative ion mode ESI mass spectrum of 4, $\alpha-1_{\mathrm{a}} \mathrm{T}_{2} 0_{\mathrm{a}} \mathrm{T}_{2} 0_{\mathrm{a}} \mathrm{T}_{2} 1_{\mathrm{a}} \mathrm{T}_{2}$. (b) MS/MS sequencing of $[4-4 \mathrm{H}]^{4-}$ at $\mathrm{m} / \mathrm{z}, 523.3$ (collision energy : $15 \mathrm{eV}$ ) and corresponding dissociation scheme. 


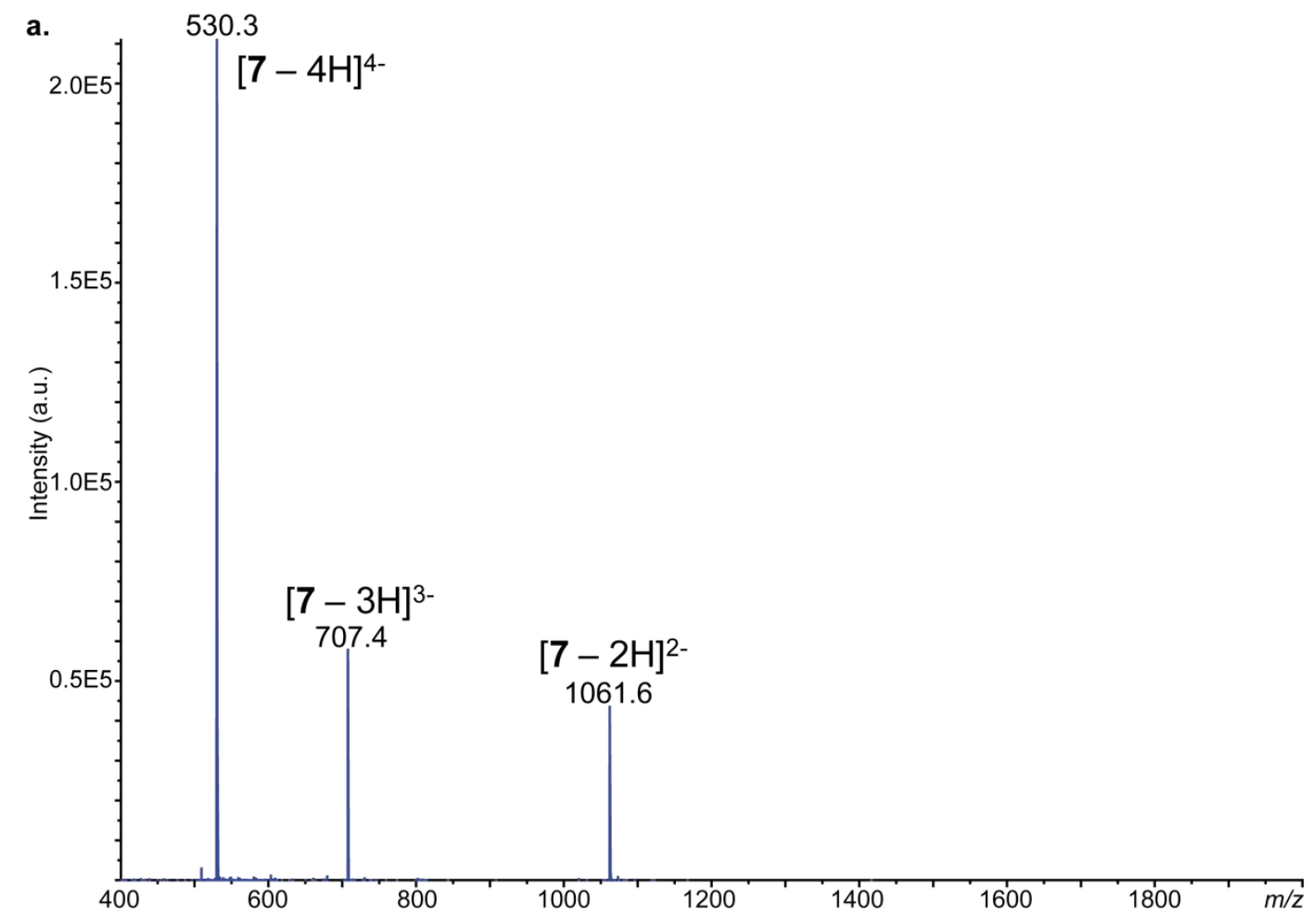

b.
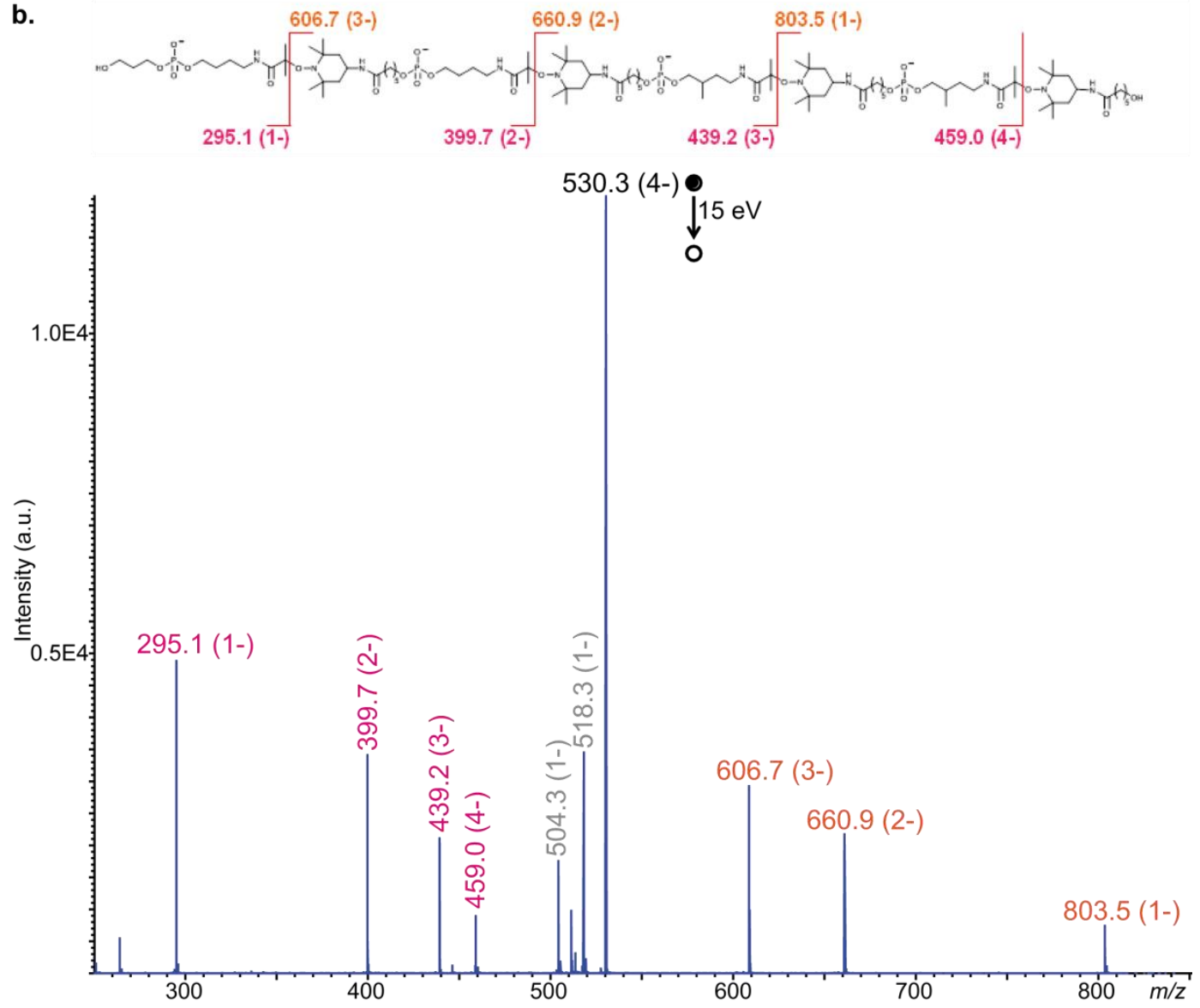

Figure S8. (a) Negative ion mode ESI mass spectrum of 7, $\alpha-0_{b} T_{2} 0_{b} T_{2} 1_{b} T_{2} 1_{b} T_{2}$. (b) MS/MS sequencing of $[7-4 \mathrm{H}]^{4-}$ at $\mathrm{m} / z 530.3$ (collision energy : $15 \mathrm{eV}$ ) and corresponding dissociation scheme. 


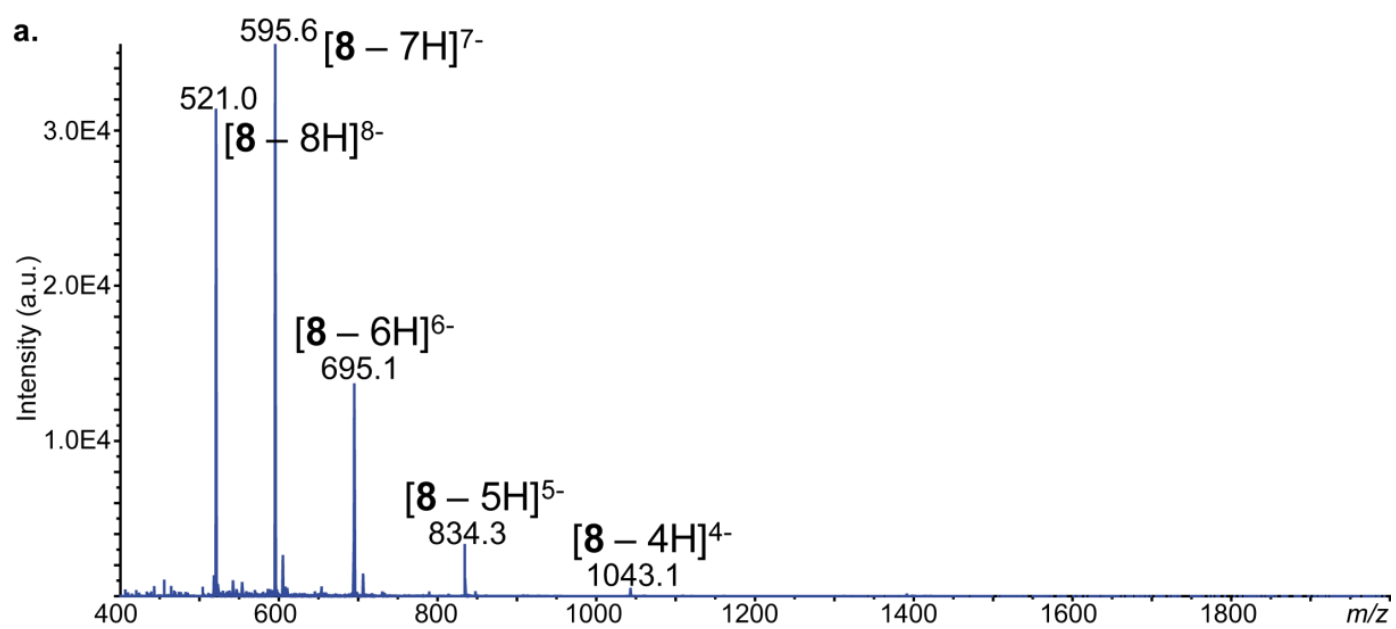

b.
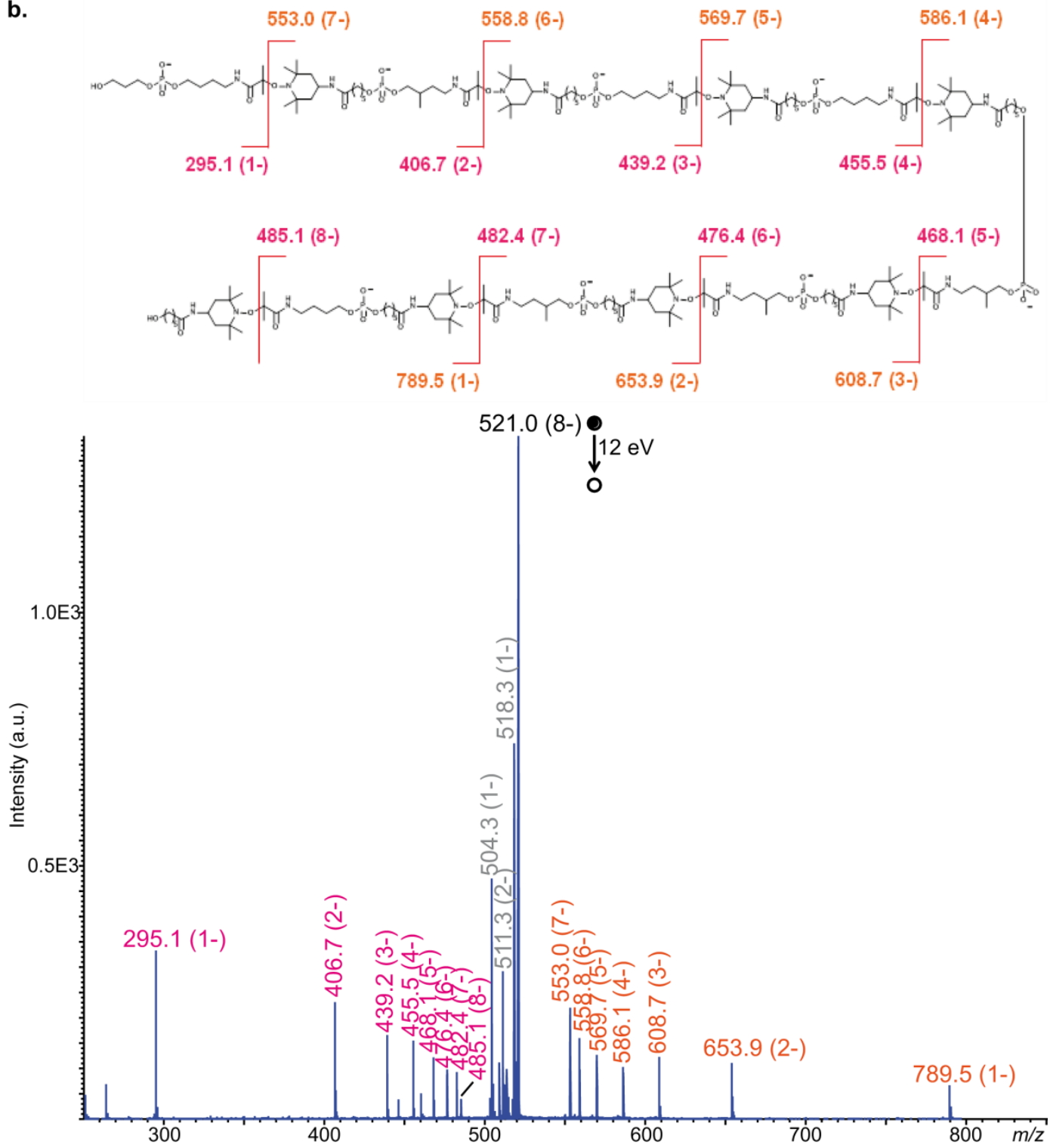

Figure S9. (a) Negative ion mode ESI mass spectrum of $8, \alpha-0_{b} T_{2} 1_{b} T_{2}\left(0_{b} T_{2}\right)_{2}\left(1_{b} T_{2}\right)_{3} 0_{b} T_{2}$. (b) MS/MS sequencing of $[8-8 \mathrm{H}]^{8-}$ at $m / z 521.0$ (collision energy: $12 \mathrm{eV}$ ) and corresponding dissociation scheme. 


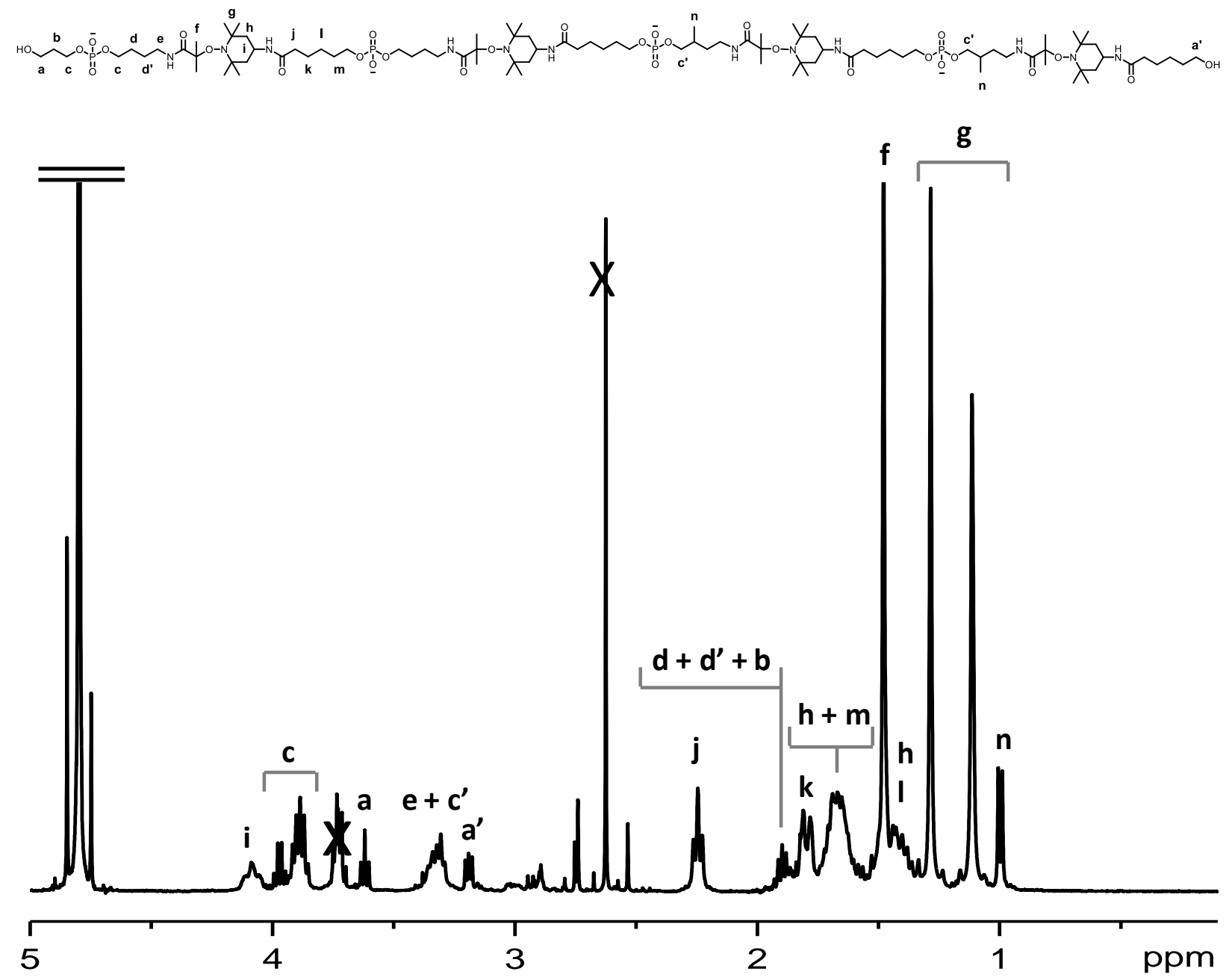

Figure S10. ${ }^{1} \mathrm{H}$ NMR spectrum recorded in $\mathrm{D}_{2} \mathrm{O}$ for copolymer $7 \alpha-0_{\mathrm{b}} \mathrm{T}_{2} 0_{\mathrm{b}} \mathrm{T}_{2} 1_{\mathrm{b}} \mathrm{T}_{2} 1_{\mathrm{b}} \mathrm{T}_{2}$. 


\section{MS/MS sequencing}

Due to their unique dissociation pattern, with homolytic cleavage of all alkoxyamine bonds occurring in a competitive manner, the sequence of any binary encoded chain can be reconstructed based on $\mathrm{m} / \mathrm{z}$ values measured for its fragments. Two series of fragments are produced, depending on the original chain end-group they still carry. When selecting the fully deprotonated molecule as the precursor ion, general structure of each series can be written (with $\mathrm{X}=0$ or 1 and the $i$ charge state being equal to the number of coding units):

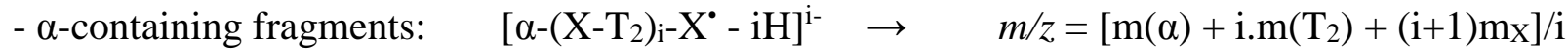

- $\omega$-containing fragments: $\quad\left[{ }^{\circ} \mathrm{T}_{2}-\left(\mathrm{X}-\mathrm{T}_{2}\right)_{\mathrm{i}}-\omega-\mathrm{i} \mathrm{H}\right]^{\mathrm{i}-} \rightarrow \quad m / z=\left[\mathrm{m}(\omega)+(\mathrm{i}+1) \mathrm{m}\left(\mathrm{T}_{2}\right)+\mathrm{i} \cdot \mathrm{m}_{\mathrm{X}}\right] / \mathrm{i}$

All possible $m / z$ values can be tabulated as a function of fragment composition, as shown in Figure S9 for precursor ions constructed with the building blocks $0_{a} / 1_{a} / T_{2}$ and containing 8 coding units (and hence activated while in the -8 charge state). All fragments listed in the two tables of Figure S9 can be distinguished based on both their $\mathrm{m} / \mathrm{z}$ values and their charge state, thanks to the resolving power of the TOF mass analyzer used here. As exemplified below with the case of $\alpha-1_{\mathrm{a}^{-}}$ $\mathrm{T}_{2}-1_{\mathrm{a}}-\mathrm{T}_{2}-0_{\mathrm{a}}-\mathrm{T}_{2}-0_{\mathrm{a}}-\mathrm{T}_{2}-0_{\mathrm{a}}-\mathrm{T}_{2}-1_{\mathrm{a}}-\mathrm{T}_{2}-0_{\mathrm{a}}-\mathrm{T}_{2}-1_{\mathrm{a}}-\mathrm{T}_{2}-\mathrm{OH}$ (data shown in Figure 3), $m / z$ values measured for all fragments (highlighted in yellow) allowed unambiguous reconstruction of the original sequence, from the left- to the right-hand side when using $\alpha$-containing fragments or from the right- to the left-hand side when using $\omega$-containing fragments.

\begin{tabular}{|c|c|c|c|c|c|c|c|c|c|c|}
\hline \multirow{2}{*}{\multicolumn{2}{|c|}{$\begin{array}{l}\text { fragments } \\
\qquad \text { with } \alpha\end{array}$}} & \multicolumn{9}{|c|}{$\mathbf{0}_{\mathbf{a}}$} \\
\hline & & 0 & I & 2 & 3 & 4 & 5 & 6 & 7 & 8 \\
\hline \multirow{9}{*}{-} & 0 & & $281.1^{\circ}$ & $385.7^{2}$ & $420.5^{3}$ & $438.0^{4-}$ & $448.4^{5-}$ & $455.4^{6-}$ & $460.4^{7-}$ & $464.1^{8}$ \\
\hline & I & $309.1^{-}$ & $399.7^{2-}$ & $429.9^{3}$ & $445.0^{4}$ & $454.0^{5-}$ & $460.1^{6-}$ & $464.4^{7-}$ & $467.6^{8-}$ & \\
\hline & 2 & $413.7^{2-}$ & $439.2^{3-}$ & $452.0^{4}$ & $459.6^{5}$ & $464.8^{6-}$ & $468.4^{7-}$ & $471.1^{8-}$ & & \\
\hline & 3 & $448.6^{3-}$ & $459.0^{4-}$ & $465.3^{5}$ & $469.4^{6}$ & $4724^{7-}$ & $474.6^{8-}$ & & & \\
\hline & 4 & $466.0^{4-}$ & $470.9^{5-}$ & $474.1^{6}$ & $476.4^{7}$ & $7478.1^{8-}$ & \\
\hline & 5 & $476.5^{5-}$ & $478.8^{6-}$ & $480.4^{7}$ & $481.6^{8}$ & \multirow{4}{*}{$\frac{\text { Sequence reconstruction }}{\text { from the left- to the right-hand side }}$} & & & & \\
\hline & 6 & $483.4^{6-}$ & $484.4^{7-}$ & $485.1^{8-}$ & & & & & & \\
\hline & 7 & $488.4^{7-}$ & $488.6^{8-}$ & & & & & & & \\
\hline & 8 & $492.2^{8-}$ & & & & & & & & \\
\hline
\end{tabular}

\begin{tabular}{|c|c|c|c|c|c|c|c|c|c|}
\hline \multirow{2}{*}{\multicolumn{2}{|c|}{$\begin{array}{l}\text { fragm ents } \\
\text { with } \omega\end{array}$}} & \multicolumn{7}{|c|}{$\mathbf{0}_{\mathbf{2}}$} & \\
\hline & & 0 & I & 2 & 3 & 4 & 5 & 6 & 7 \\
\hline \multirow{8}{*}{$=$} & 0 & & $775.5^{\circ}$ & $632.9^{2-}$ & $585.3^{3-}$ & $561.6^{4-}$ & $547.3^{5-}$ & $537.8^{6-}$ & $531.0^{7-}$ \\
\hline & I & 8035 & $6469^{2-}$ & $594.7^{3-}$ & $568.5^{4-}$ & $552.9^{5-}$ & $542.5^{6-}$ & $535.0^{7-}$ & \\
\hline & 2 & $660.9^{2-}$ & $604 d^{3-}$ & $575.6^{4}$ & $5585^{5}$ & $547.1^{6-}$ & $539.0^{7-}$ & & \\
\hline & 3 & $613.4^{3-}$ & $582.6^{4-}$ & $564.1^{5-}$ & $551.8^{6-}$ & $543.0^{7}$ & \\
\hline & 4 & $589.6^{4-}$ & $569.7^{5}$ & $556.5^{6-}$ & $547.0^{7-}$ & \multirow{4}{*}{$\begin{array}{l}\frac{\text { Sequence reconstruction }}{\text { from the right- to the left-hand side }} \\
\mathrm{T}_{2} \mathbf{0}_{\mathrm{a}} \mathrm{T}_{2} \mathbf{0}_{\mathrm{a}} \mathrm{T}_{2} \mathbf{0}_{\mathrm{a}} \mathrm{T}_{2} \mathbf{l}_{\mathrm{a}} \mathrm{T}_{2} \mathbf{0}_{\mathrm{a}} \mathrm{T}_{2} \mathbf{l}_{\mathrm{a}}-\omega\end{array}$} & & & \\
\hline & 5 & $575.3^{5-}$ & $561.2^{6-}$ & $551.0^{7-}$ & & & & & \\
\hline & 6 & $565.8^{6-}$ & $555.0^{7-}$ & & & & & & \\
\hline & 7 & $559.0^{7-}$ & & & & & & & \\
\hline
\end{tabular}

Figure S11. Tabulated $\mathrm{m} / \mathrm{z}$ values (with superscripted charge state) of all fragments expected from any precursor ion containing 8 coding units and activated as a $[\mathrm{M}-8 \mathrm{H}]^{8-}$ ion. Italicized values indicate the number of $\mathbf{0}_{\mathrm{a}}$ or $\mathbf{1}_{\mathrm{a}}$ coding units in a given fragment. Calculation based on $\mathrm{m}_{\alpha}=59.0$ $\mathrm{Da}, \mathrm{m}_{\omega}=17.0 \mathrm{Da}$, and building block residues: $\mathrm{m}\left(0_{\mathrm{a}}\right)=222.1 \mathrm{Da}, \mathrm{m}\left(1_{\mathrm{a}}\right)=250.1 \mathrm{Da}$, and $\mathrm{m}\left(\mathrm{T}_{2}\right)=$ 268.2 Da. 


\section{References}

[1] Al Ouahabi, A.; Charles, L.; Lutz, J.-F., J. Am. Chem. Soc., 2015,137, 5629-5635.

[2] Averick, S. E.; Dey, S. K.; Grahacharya, D.; Matyjaszewski, K.; Das, S. R. Angew. Chem., Int. Ed., 2014, 53, $2739-2744$.

[3] Couet, W.R.; Brasch, R.C.; Sosnovsky, C.; Lukszo, J.; Prakash, I.; Gnewech, C.T.; Tozer, T.N., Tetrahedron, 1985, 41, 1165-1172. 